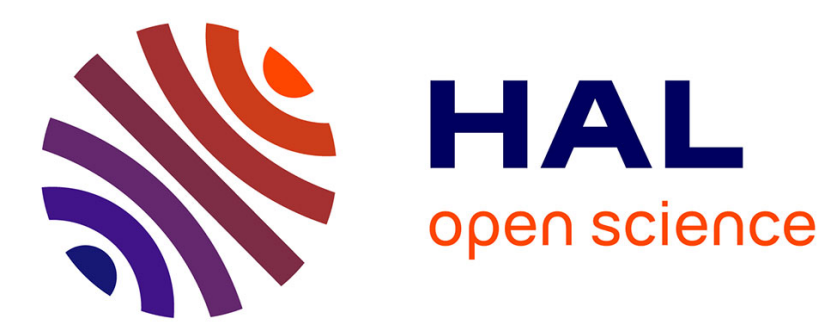

\title{
Behavior of poroelastic isotropic beam derivation by asymptotic expansion method
}

\author{
Claude Boutin
}

\section{To cite this version:}

Claude Boutin. Behavior of poroelastic isotropic beam derivation by asymptotic expansion method. Journal of the Mechanics and Physics of Solids, 2012, 60 (6), pp.1063-1087. hal-00943726

\section{HAL Id: hal-00943726 \\ https://hal.science/hal-00943726}

Submitted on 8 Feb 2014

HAL is a multi-disciplinary open access archive for the deposit and dissemination of scientific research documents, whether they are published or not. The documents may come from teaching and research institutions in France or abroad, or from public or private research centers.
L'archive ouverte pluridisciplinaire HAL, est destinée au dépôt et à la diffusion de documents scientifiques de niveau recherche, publiés ou non, émanant des établissements d'enseignement et de recherche français ou étrangers, des laboratoires publics ou privés. 


\title{
Behavior of poroelastic isotropic beam derivation by asymptotic expansion method
}

\section{Claude Boutin}

Université de Lyon, Ecole Nationale des Travaux Publics de PEtat, DGCB CNRS 3237 Rue Maurice Audin, 69518 Vaulx-en-Velinn, France

\begin{abstract}
A B S T R A C T
This paper deals with the dynamic behavior of poroelastic beams, i.e. rod made of a biphasic medium described by the Biot model. The study mostly focuses on situations where the inner flow is dominated by viscosity, but also investigate the case of viscoinertial inner flow. Using the inverse of the slenderness as a small parameter, one establishes through asymptotic expansions the 1D beam description in harmonic regime: the Euler-Bernoulli kinematic still applies, however the equilibrium of the section induces a poroelastic problem with pressure diffusion. The beam parameters are rigorously derived from this problem and can be computed numerically. They are complex and frequency dependent which implies creep/relaxation mechanisms. This theoretical formulation is discussed according to the level of permeability, the flow conditions on the section periphery, the gas or liquid nature of the fluid, the frequency range of the oscillations. Analytical and numerical results are provided for circular and flat beam sections.
\end{abstract}

\section{Introduction}

Number of 1D structures encountered in biomechanics (bones, plant stems, trunks, roots) or 2D structures common in geomechanics (draining layer) and in acoustics (isolating foam plates) can be considered as poroelastic beams or plates in the sense that bending is a driving phenomenon. It has been recognized from the works of Taber (1992) and Scherer (1992) that bending deformation of poroelastic plates induces a non-uniform pressure distribution within the section. This latter is associated with a diffusion mechanism that induces creep and relaxation phenomena. As for poroelastic beams, two situations have been investigated: either the material is isotropic and a mechanism of lateral diffusion may occur, cf. Zhang and Cowin (1994) and Scherer et al. (2009), or the media is anisotropic and enables diffusion in the axial direction only (Li et al., 1995). In the isotropic case, the complexity of the poroelastic stress-strain distribution in the beam has led to develop approximated solutions. These latter are built phenomenologically by extension of the classical elastic beam theory, assuming a zero total stress tensor in the plane of the section, or considering specific boundary condition to insure a single direction of the flow. In the above-mentioned works, the frequency range is assumed to be sufficiently low, so that the dynamic permeability lies in the viscous regime.

The purpose of this paper is to derive the quasi-static and dynamic behavior of poroelastic isotropic beams and to identify the domain of validity of the existing approximations. In the framework of elasticity, Trabucho and Viano (1996) have shown that, accounting for the geometrical features of slender bodies, the asymptotic methods enable to move from the $3 \mathrm{D}$ constitutive law of the material to the $1 \mathrm{D}$ beam behavior. This approach is reconsidered here in the framework of 
dynamic poroelasticity, in the case of homogeneous straight beams made of isotropic material. The compression and bending beam parameters are rigorously derived. They can either be computed, estimated under simplifying assumption or even formulated analytically for beam of circular section. The procedure enables to specify clearly the different regimes of behavior, according to the mechanical parameters of the porous material, the compressibility of the saturating fluid, the nature of the flow boundary conditions on the beam periphery, the frequency range of oscillations and the viscous or visco-inertial character of the fluid flow at the pore level.

The paper is organized as follows. Section 2 briefly recalls the description of saturated poroelastic materials and introduces the poroelastic beam problem. Section 3 is devoted to the physical analysis of the equilibrium of slender poroelastic bodies, when the inner flow regime is dominated by viscosity. In Section 4, the poroelastic beam behavior is derived through the asymptotic expansion method. The results are discussed in Section 5 and illustrated in Section 6 where an exact poroelastic description is given for beams of flat and of circular section. The situation of visco-inertial flow is addressed in Section 7.

\section{Setting of the poroelastic beam problem}

\subsection{Governing equations of the poroelastic material}

The pore sizes of the medium are assumed to be much smaller than the dimension $h$ of the beam section and the viscosity of the saturating fluid is low enough to allow fluid displacement relatively to the solid. This enables to adopt the phenomenological Biot (1956) description (see also Coussy, 2004) in the form derived by homogenization (SanchezPalencia, 1980; Auriault, 1980) (see also Auriault et al., 2009). Thus, considering isotropic poroelastic materials the behavior of the saturated porous material is governed by the following set ( $\cdot$ stands for time derivative, and $*$ for time convolution):

$$
\begin{aligned}
& \underline{\operatorname{div}}(\underline{\underline{\Sigma}})=(1-\phi) \rho_{\mathrm{s}} \underline{\ddot{u}}+\phi \rho_{\mathrm{f}} \underline{\ddot{u}}_{\mathrm{f}} \\
& \underline{\underline{\Sigma}}=\underline{\underline{\sigma}}-\alpha p \underline{\underline{\mathrm{I}}}, \quad \underline{\underline{\sigma}}=\lambda \operatorname{div}(\underline{u}) \underline{\underline{\mathrm{I}}}+2 \mu \underline{\underline{\mathbf{e}}}(\underline{u}) \\
& \phi \operatorname{div}\left(\underline{\dot{u}}_{\mathrm{f}}-\underline{\dot{u}}\right)=-\alpha \operatorname{div}(\underline{\dot{u}})-\frac{\dot{p}}{M}, \\
& \phi\left(\underline{\dot{u}}_{\mathrm{f}}-\underline{\dot{u}}\right)=-\frac{\mathbf{K}(\tau)}{\eta} *\left[\rho_{\mathrm{f}} \underline{\ddot{u}}+\underline{\operatorname{grad}}(p)\right] .
\end{aligned}
$$

These equations express the dynamic equilibrium (1), the conservation of fluid mass ( 3 ), the poroelastic constitutive law (2) and the dynamic Darcy's law (4) with the following notations:

- $\phi$ is the porosity, $\rho_{\mathrm{s}}, \rho_{\mathrm{f}}$ and $\rho_{m}=(1-\phi) \rho_{\mathrm{s}}+\phi \rho_{\mathrm{f}}$ are the densities of the materials forming the porous skeleton, the saturating fluid and the saturated porous medium.

- $\alpha=1-K_{b} / K_{\mathrm{s}}$ is Biot's coefficient $(\phi \leq \alpha \leq 1)$;

$$
\frac{1}{M}=\frac{\alpha-\phi}{K_{\mathrm{s}}}+\frac{\phi}{K_{\mathrm{f}}}
$$

is Biot's bulk modulus. In these expressions, $K_{\mathrm{s}}, K_{b}$ and $K_{\mathrm{f}}$ are respectively the bulk moduli of the material forming the porous media, of the empty (or drained) porous media and of the fluid.

- $\lambda$ and $\mu$ stand for the Lamé coefficients of the dry porous media, $E=2 \mu(1+v)$ for its Young modulus and $v=\lambda / 2(\lambda+\mu)$ for the Poisson ratio $(\lambda+2 \mu=2(1-v)(\lambda+\mu))$. We will also use the "consolidation" modulus $A_{c}$ defined by

$$
\frac{1}{A_{c}}=\frac{1}{M}+\frac{\alpha^{2}}{\lambda+2 \mu}
$$

and the modulus $A$ defined by

$$
\frac{1}{A}=\frac{1}{M}+\frac{\alpha^{2}}{\lambda+\mu} \text {. }
$$

- $\underline{u}$ and $\underline{u}_{\mathrm{f}}$ are the solid and fluid displacements (i.e. the mean displacement over the volume of the pores). The Darcy velocity is $\phi\left(\underline{\dot{u}}_{\mathrm{f}}-\underline{\dot{u}}\right)$.

- $\underline{\underline{\mathbf{e}}}(\underline{u})$ is the strain tensor; $\Sigma, \sigma$ and $p$ represent respectively the tensor of total stress of effective stress (i.e. mean stress in the solid skeleton) and the interstitial pressure.

- $\eta$ is the fluid viscosity and $\mathbf{K}(\tau)$ is the impulse flux response or the memory permeability function (in units of $\mathrm{m}^{2}$ ). Its Fourier transform $K(\omega)$ defines the dynamic permeability. 
Under harmonic oscillations regime of frequency $f=\omega / 2 \pi$, the above governing equations take the form (by linearity of the problem we can ignore the time-dependent term $e^{i \omega t}$ in all the equations):

$$
\begin{aligned}
& \underline{\operatorname{div}}(\underline{\underline{\Sigma}})=-\omega^{2}\left[(1-\phi) \rho_{\mathrm{s}} \underline{u}+\phi \rho_{\mathrm{f}} \underline{u}_{\mathrm{f}}\right], \\
& \underline{\underline{\Sigma}}=\underline{\underline{\sigma}}-\alpha p \underline{\underline{\mathrm{I}}}, \quad \underline{\underline{\sigma}}=\lambda \operatorname{div}(\underline{u}) \underline{\underline{\mathrm{I}}}+2 \mu \underline{\underline{\mathbf{e}}}(\underline{u}), \\
& \phi \operatorname{div}\left(\underline{u}_{\mathrm{f}}-\underline{u}\right)=-\alpha \operatorname{div}(\underline{u})-\frac{p}{M}, \\
& i \omega \phi\left(\underline{u}_{\mathrm{f}}-\underline{u}\right)=\frac{\mathrm{K}(\omega)}{\eta}\left[\omega^{2} \rho_{\mathrm{f}} \underline{u}-\underline{\operatorname{grad}}(p)\right] .
\end{aligned}
$$

Following Auriault et al. (1985), at low frequencies $(\omega \rightarrow 0)$ viscous effects dominate and $\mathrm{K}(\omega) \rightarrow \mathrm{K}(0)=\mathcal{K}$, where $\mathcal{K}$ is the intrinsic permeability that is of the order of the square of the typical size of the pores; at high frequencies $(\omega \rightarrow \infty)$ inertia dominates: $i \omega \rho_{\mathrm{f}} \mathrm{K}(\omega) / \eta \rightarrow \phi / \tau_{\infty}$, this leads to an "additional mass" effect quantified by the tortuosity $\tau_{\infty} \geq 1$.

The transition from low to high-frequency occurs around the characteristic frequency $f_{c}=\omega_{c} / 2 \pi$ where the viscous terms, estimated by the low-frequency approximation, are of the same order as the inertial terms, using the highfrequency approximation; hence:

$$
\omega_{c}=\frac{\phi \eta}{\mathrm{K}(0) \rho_{\mathrm{f}} \tau_{\infty}}=\frac{\phi \eta}{\mathcal{K} \rho_{\mathrm{f}} \tau_{\infty}}
$$

A simple analytical form respecting the asymptotic features of $K(\omega)$ has been proposed by Johnson et al. (1987); expressions are also available for packing of spheres and polyhedrons (Boutin and Geindreau, 2010).

\subsection{The (뜨,p) poroelastic formulation}

The set (5)-(8) links the total stress and the interstitial pressure to the fields of the solid and fluid displacements. However, the solid motion $\underline{\mathrm{u}}$ and the pressure $p$ are enough to describe the porous medium. The so-called $(\underline{\mathrm{u}}, p)$ poroelastic formulation is derived by eliminating $\underline{u}_{f}$ and $\Sigma$ in (5)-(8). This provides four scalar conservation equations (three in force, one in mass) describing the porous medium with the four independent scalar variables $\left\{u_{i}, p\right\}$ :

$$
\begin{aligned}
& (\lambda+\mu) \underline{\operatorname{grad}}(\operatorname{div}(\underline{u}))+\mu \Delta(\underline{u})-\left(\alpha-\frac{i \omega \rho_{\mathrm{f}} \mathrm{K}}{\eta}\right) \underline{\operatorname{grad}}(p)=-\omega^{2}\left[\rho_{m}+\frac{i \omega \rho_{\mathrm{f}} \mathrm{K}}{\eta} \rho_{\mathrm{f}}\right] \underline{u} \\
& \operatorname{div}\left(\frac{\mathrm{K}}{i \omega \eta} \underline{\operatorname{grad}}(p)\right)-\left(\alpha-\frac{i \omega \rho_{\mathrm{f}} \mathrm{K}}{\eta}\right) \operatorname{div}(\underline{u})=\frac{p}{M}
\end{aligned}
$$

Eq. (9) is similar to an elasto-dynamic equation with a coupling term of pressure gradient, and Eq. (10) is similar to a transient diffusion equation of pressure with a coupling term of solid volume variation. Note the symmetry of the $(\underline{u}, p)$ coupling.

In quasi-statics, the inertia induced by the whole motion is negligible, i.e. $-\omega^{2} \rho_{m} \underline{u} \rightarrow 0$, and the pores scale flow is dominated by viscosity regime, hence the pores scale flow inertia vanishes, i.e. $i \omega \rho_{\mathrm{f}} \mathrm{K} / \eta \rightarrow 0$, or $\omega / \omega_{c} \ll 1$. This leads to the usual quasi-static version of (9)-(10) where only elastic and viscous effects are taken into account:

$$
\begin{aligned}
& \underline{\operatorname{div}}(\underline{\underline{\Sigma}})=(\lambda+\mu) \underline{\operatorname{grad}}(\operatorname{div}(\underline{u}))+\mu \Delta(\underline{u})-\alpha \underline{\operatorname{grad}}(p)=\underline{0} \\
& \operatorname{div}\left(\frac{\mathcal{K}}{i \omega \eta} \underline{\operatorname{grad}}(p)\right)-\alpha \operatorname{div}(\underline{u})=\frac{p}{M}
\end{aligned}
$$

The derivation of the poroelastic beam behavior will be performed in this framework, using (11)-(12). However, in some cases, the frequency range of both inertia effects may significantly differ, and visco-inertial flow can arise at the pore scale, i.e. $i \omega \rho_{\mathrm{f}} \mathrm{K} / \eta=O(1)$, while the whole motion inertia remains negligible, i.e. $\omega^{2} \rho_{m} \underline{u} \rightarrow 0$. This particular situation of viscoinertial flow regime is treated in Section 7.

\subsection{Poroelastic boundary conditions}

The conditions at the boundary of a poroelastic medium can be the continuity of solid displacement, of flux, of total stress and of pressure (all conditions can be expressed in terms of (u, $p$ ) variables through the poroelastic constitutive law, and the dynamic Darcy law).

For rods unloaded on their current section (Fig. 1), with a perfectly pervious surface $\Gamma$ (of normal $\underline{n}$ ), the boundary conditions read

$$
\begin{aligned}
& \underline{\underline{\Sigma}} \cdot \underline{\underline{n}}=[\lambda \operatorname{div}(\underline{u}) \underline{\underline{I}}+2 \mu \underline{\underline{\mathbf{e}}}(\underline{u})] \cdot \underline{n}-\alpha p \underline{\underline{n}}=\underline{0} \\
& p=0
\end{aligned}
$$




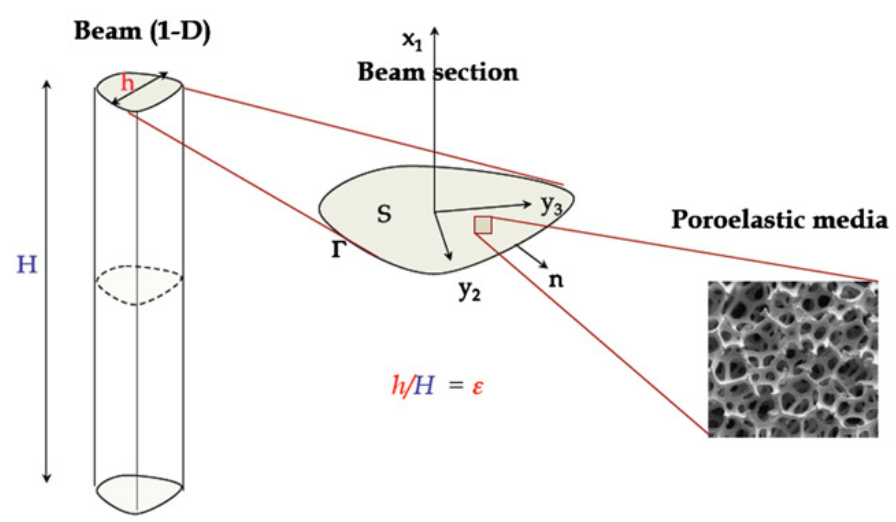

Fig. 1. Beam made of poroelastic material.

while, in case of perfectly impervious surface, we have instead of (14):

$$
\phi i \omega\left(\underline{u}_{\mathrm{f}}-\underline{u}\right) \cdot \underline{n}=\frac{\mathrm{K}(\omega)}{\eta}\left[\omega^{2} \rho_{\mathrm{f}} \underline{u}-\underline{\operatorname{grad}}(p)\right] \cdot \underline{n}=0
$$

For more generality the perfectly pervious or impervious condition, can be replaced by a leakage boundary condition: assuming the porous beam surface covered by a thin layer of thickness $e$ and permeability $k$, the pressure drop from the beam $(p)$ to the external surface $(p=0)$ is related to the normal flux by $v=-(k / \eta)(p / e)$. From the flux continuity and denoting $k h / K e=\xi$ ( $h$ is the characteristic size of the beam section) we obtain the following leakage condition:

$$
\frac{\xi}{h} p=\phi i \omega\left(\underline{u}_{\mathrm{f}}-\underline{u}\right) \cdot \underline{n} \frac{\eta}{\mathrm{K}(\omega)} \quad \text { i.e. in quasi }- \text { statics : } \frac{\xi}{h} p=-\underline{\operatorname{grad}}(p) \cdot \underline{n}
$$

The dimensionless surface leakage coefficient $\xi$ enables to treat boundaries neither perfectly pervious, that would correspond to $\xi=\infty$, nor perfectly impervious, $\xi=0$.

Remark. Other type of condition could also be introduced, for instance when a thin elastic film is coated on the surface. The film behaves as a deformable membrane fixed on the porous matrix. Hence, the fluid crossing the pores section $\phi\left(\underline{u}_{\mathrm{f}}-\underline{u}\right) \cdot \underline{n}$ induces a proportional tension in the membrane, that is balanced by the effective stress on the matrix, i.e. $\phi\left(\underline{u}_{\mathrm{f}}-\underline{u}\right) \cdot \underline{\underline{n}} \sim \underline{n} \cdot \underline{\underline{\sigma}} \cdot \underline{n}=\alpha p$ (since the total stress vanishes). Thus, in this configuration the boundary condition would read in quasi-statics, where $\kappa$ is proportional to the membrane rigidity:

$$
\alpha p=-\frac{\kappa}{h} \frac{\mathcal{K}}{i \omega \eta} \underline{\operatorname{grad}}(p) \cdot \underline{n}
$$

This condition tends to impervious boundary at sufficiently low frequency, and to free flow at sufficiently large frequency.

\subsection{Balance equations of forces and momentum in poroelastic beams}

A rod is characterized by the fact that the axial dimension $L$ is significantly larger than the typical dimension $h$ of the sectionS (Fig. 1). A direct consequence of this slender geometry is the specificity of the axial direction. This leads to split any tensor $\underline{\underline{A}}=A_{i j}\left(\underline{e}_{i} \otimes \underline{e}_{j}+\underline{e}_{j} \otimes \underline{e}_{i}\right) / 2(\underline{\underline{A}}=\underline{\underline{\Sigma}}$ for total stress; $\underline{\underline{A}}=\underline{\underline{\sigma}}$ for effective stress; $\underline{\underline{A}}=\underline{\underline{e}}$ for strain) into reduced tensors (here and in the sequel $\underline{a}_{1}$ denotes the axial direction, $\underline{a}_{\alpha}$ the directions in the plane of the section; Greek and Latin indices respectively run from 2 to 3 , and 1 to 3 ):

$$
\underline{\underline{A}}=A_{\mathrm{n}} \underline{a}_{1} \otimes \underline{a}_{1}+\left(\underline{A}_{\mathrm{t}} \otimes \underline{a}_{1}+\underline{a}_{1} \otimes \underline{A}_{\mathrm{t}}\right) / 2+\underline{\underline{A}}_{\mathrm{s}}
$$

where $A_{\mathrm{n}}=A_{11}$ is the scalar axial stress or strain, $\underline{A}_{\mathrm{t}}=A_{1 \alpha} \underline{a}_{\alpha}$ is the vector of the stress or strain exerted out of the plane of the section, $\underline{\underline{A}}_{S}=A_{\alpha \beta}\left(\underline{a}_{\alpha} \otimes \underline{e}_{\beta}+\underline{e}_{\beta} \otimes \underline{a}_{\alpha}\right) / 2$ is the second rank tensor of the stress or strain in the plane of the section.

With these notations the balance equation (11) and the boundary conditions (13) may be split into the following axial and in-plane balance $\left(, x_{1}, \operatorname{div}_{x_{\alpha}}, \ldots\right.$ stands respectively for the derivative according to $x_{1}$, for the in-plane divergence, ...):

$$
\begin{aligned}
& \sum_{\mathrm{n}, x_{1}}+\operatorname{div}_{x_{\alpha}}\left(\underline{\sigma}_{\mathrm{t}}\right)=0 \text { in } S \\
& \underline{\sigma}_{\mathrm{t}} \cdot \underline{n}=0 \quad \text { on } \Gamma \\
& \underline{\sigma}_{\mathrm{t}, x_{1}}+\underline{\operatorname{div}}_{x_{\alpha}}\left(\underline{\underline{\Sigma}}_{\mathrm{s}}\right)=\underline{0} \text { in } S \\
& \underline{\underline{\Sigma}}_{\mathrm{s}} \cdot \underline{n}=\underline{0} \text { on } \Gamma
\end{aligned}
$$


The balance equations of global forces acting on the section are derived by integrating (17) and (19) over $S$. The divergence theorem and boundary conditions (18)-(20) yield:

$$
\int_{S} \operatorname{div}_{x_{\alpha}}\left(\underline{\sigma}_{\mathrm{t}}\right) d s=\int_{\Gamma} \underline{\sigma}_{\mathrm{t}} \cdot \underline{n} d \gamma=0 \text { and } \int_{S} \underline{\operatorname{div}}_{x_{\alpha}}\left(\underline{\underline{\Sigma}}_{\mathrm{S}}\right) d s=\int_{\Gamma} \underline{\underline{\Sigma}}_{\mathrm{s}} \cdot \underline{n} d \gamma=\underline{0}
$$

Thus, inverting $x_{\alpha}$-integration and $x_{1}$-derivate provides the following balance equations over the section (valid when the beam is free of any surface and volume loading):

$$
\text { along } \underline{a}_{1}: \frac{\partial}{\partial x_{1}}\left(\int_{S} \Sigma_{\mathrm{n}} d s\right)=0 \text {, along } \underline{e}_{2}, \underline{e}_{3}: \frac{\partial}{\partial x_{1}}\left(\int_{S} \underline{\sigma}_{\mathrm{t}} d s\right)=\underline{0}
$$

Three global momentum equilibrium equations are also established considering separately the axial and in-plane directions. First, multiply (17) by $x_{\alpha}$ and integrate over $S$ :

$$
\int_{S} x_{\alpha} \Sigma_{\mathrm{n}, x_{1}} d s+\int_{S} x_{\alpha} \operatorname{div}_{x_{\alpha}}\left(\underline{\sigma}_{\mathrm{t}}\right) d s=0
$$

Integrating the second integral by part and applying the divergence theorem yields:

$$
\int_{S} \operatorname{div}_{x_{\alpha}}\left(x_{\alpha} \underline{\sigma}_{\mathrm{t}}\right) d s-\int_{S} \underline{\sigma}_{\mathrm{t}} \cdot \underline{a}_{\alpha} d s=\int_{\Gamma} x_{\alpha}\left(\underline{\sigma}_{\mathrm{t}} \cdot \underline{\underline{n}}\right) d \gamma-\int_{S} \underline{\sigma}_{\mathrm{t}} \cdot \underline{a}_{\alpha} d s
$$

and the integral over $\Gamma$ vanishes because of the free boundary condition (18). Finally, inverting $x_{\alpha}$-integration and $x_{1}$ derivate leads to the two momentum of momentum balance equations:

$$
\text { along } \underline{a}_{\alpha}: \frac{\partial}{\partial x_{1}}\left(\int_{S} x_{\alpha} \Sigma_{\mathrm{n}} d s\right)-\int_{S} \underline{\sigma}_{\mathrm{t}} \cdot \underline{a}_{\alpha} d s=0
$$

The global momentum of momentum balance in direction $\underline{a}_{1}$ is established by taking the vectorial product of (26) by the position vector $\underline{x}=x_{\alpha} \cdot \underline{a}_{\alpha}$ and integrating over the section:

$$
\int_{S} \underline{x} \times \underline{\sigma}_{\mathrm{t}, x_{1}} d s+\varepsilon^{-1} \int_{S} \underline{x} \times \underline{\operatorname{div}}_{x_{\alpha}}\left(\underline{\underline{\Sigma}}_{\mathrm{s}}\right) d s=\underline{0}
$$

The second integral reads ( $\epsilon$ is the third rank tensor expressing the vectorial product):

$$
\underline{a}_{1}\left[\int_{S} \epsilon_{1 \alpha \beta} x_{\alpha} \Sigma_{\beta \gamma, y_{\gamma}} d s\right]=\underline{a}_{1}\left[\int_{S} \epsilon_{1 \alpha \beta} x_{\alpha} \sigma_{\beta \gamma, x_{\gamma}} d s\right]
$$

Integrating by part, then using the divergence theorem and the symmetry of $\underline{\underline{\Sigma}}$, and finally the free boundary condition (20), show that this term vanishes:

$$
\int_{S} \epsilon_{1 \alpha \beta} x_{\alpha} \sigma_{\beta \gamma, x_{\gamma}} d s=\int_{\Gamma} \underline{x} \times\left(\underline{\underline{\sigma}}_{s} \cdot \underline{n}\right) d \Gamma=0
$$

Consequently, along $\underline{a}_{1}$ :

$$
\frac{\partial}{\partial x_{1}}\left(\int_{S} \underline{x} \times \underline{\sigma}_{\mathrm{t}} d s\right)=\underline{0}
$$

To sum up, denoting by $N \underline{a}_{1}$ and $\underline{T}=T_{\alpha} \underline{a}_{\alpha}$ the normal and shear forces, and by $\underline{M}=M_{\alpha} \underline{a}_{\alpha}$ and $M_{1} \underline{a}_{1}$ the bending and torsion momentum respectively the balance equations of beams free of surface and volume loading are

$$
\begin{aligned}
& \text { along } \underline{a}_{1}: \frac{\partial N}{\partial x_{1}}=0, N=\int_{S} \Sigma_{\mathrm{n}} d s, \quad \frac{\partial M_{1}}{\partial x_{1}}=0, M_{1}=\underline{a}_{1} \cdot \int_{S} \underline{x} \times \underline{\sigma}_{\mathrm{t}} d s \\
& \text { along } \underline{a}_{\alpha}: \frac{\partial \underline{M}}{\partial x_{1}}-\underline{T}=\underline{0}, \underline{M}=\int_{S} \underline{x} \Sigma_{\mathrm{n}} d s, \quad \frac{\partial \underline{T}}{\partial x_{1}}=\underline{0}, \underline{T}=\int_{S} \underline{\sigma}_{\mathrm{t}} d s
\end{aligned}
$$

Remark. A similar treatment could be applied to the mass balance. However, this is unnecessary since we will show that the pressure in the beam is not an independent variable, but a variable driven by the solid motions.

To go further, it is necessary to relate the forces and momentum to the solid motion (and the pressure). This is achieved in the following sections by means of scaling and asymptotic expansions. We will establish the following constitutive poroelastic beam laws that constitute the main result of the paper (see Section 4 for notations):

$$
\begin{aligned}
& N=\widehat{E S} \frac{\partial U_{1}}{\partial x_{1}}, \quad \widehat{E S}=E|S|-[(1-2 v) \alpha]^{2} A \int_{S} \zeta d S \\
& M_{\alpha}=-\widehat{E I_{\alpha}} \frac{\partial^{2} U_{\alpha}}{\partial x_{1}^{2}}, \quad \widehat{E I_{\alpha}}=E I_{\alpha}+[(1-2 v) \alpha]^{2} A \int_{S} x_{\alpha} \psi^{\alpha} d S \\
& M_{1}=\mu I_{t} \frac{\partial \Omega}{\partial x_{1}}
\end{aligned}
$$




\section{Scaling of the physics of poroelastic rod}

The purpose of this section is to express mathematically the consequences of the slender geometry, and to specify the physics through a scaling process. This is addressed considering homogeneous straight beams made of isotropic poroelastic material and assuming the current beam section free of surface and volume forces. This formulation enables the development of the asymptotic method presented in Section 4, that leads to the complete beam description under quasi-static or dynamic loading.

\subsection{Usual and scaled variables}

The inverse of the slenderness naturally defines the small parameter $\varepsilon=h / L \ll 1$, used in the expansions. Moreover, the dimensionless spaces variables reflecting the characteristic sizes along $\underline{a}_{1}$ and $\underline{a}_{\alpha}$ are $\left(x_{1} / L, x_{2} / h, x_{3} / h\right)$ (see Fig. 1). Equivalently, $\left(x_{1}, y_{2}, y_{3}\right)$, where $y_{\alpha}=(L / h) x_{\alpha}=\varepsilon^{-1} x_{\alpha}$ are the appropriate physical space variables. The usual gradient $\underline{\nabla}=\partial_{x_{i}} \underline{e}_{i}$ - that applies on $\varphi(\underline{x})$ - becomes for the same quantity $\varphi$ expressed with $\left(x_{1}, y_{\alpha}\right)$ :

$$
\underline{\nabla} \varphi=\left(\partial_{x_{1}} \underline{a}_{1}+\varepsilon^{-1} \partial_{y_{\alpha}} \underline{a}_{\alpha}\right) \varphi\left(x_{1}, \underline{y}\right)
$$

Similarly, the integrals are modified as $\left(d s=d y_{2} d y_{3} ; d \gamma=d y_{\Gamma}\right)$ :

$$
\int_{S} \varphi(\underline{x}) d S=\varepsilon^{2} \int_{S} \varphi\left(x_{1}, \underline{y}\right) d s, \quad \int_{\Gamma} \varphi(\underline{x}) d \Gamma=\varepsilon \int_{\Gamma} \varphi\left(x_{1}, \underline{y}\right) d \gamma
$$

and we will use the following notations:

$$
|S|=\int_{S} d x_{2} d x_{3},\left|S^{\prime}\right|=\int_{S} d y_{2} d y_{3}=\varepsilon^{-2}|S|, \quad I_{\alpha}=\int_{S} x_{\alpha}^{2} d x_{2} d x_{3}, I_{\alpha}^{\prime}=\int_{S} y_{\alpha}^{2} d y_{2} d y_{3}=\varepsilon^{-4} I_{\alpha}
$$

In the sequel the problems are formulated in the main $y$-frame, i.e. the frame originated at the center of "mass" of section $S$ and orientated along its principal axis of inertia. Thus:

$$
\int_{S} y_{\alpha} d s=0, \quad \int_{S} y_{\alpha} y_{\beta} d s=0 \text { for } \alpha \neq \beta
$$

Sections that present two orthogonal axis of symmetry will be referred as bi-symmetric. Finally, descriptions will be designated as scaled when based on variables $\left(x_{1}, y_{\alpha}\right)$, and as usual when using variables $\left(x_{1}, x_{\alpha}\right)$ (i.e. same length units in the three directions, as in usual practice).

\subsection{Beam kinematics and reduced tensors}

Two facts constrain the beam kinematics: first, the geometry of straight, homogeneous, unloaded beam suggests that the phenomena vary along the axis according to $L$ and within the section according to $h$; second, the absence of tangential forces on the contour $\Gamma$. Hence, denoting the normal of the straight beam boundary $\Gamma$ by $\underline{n}=n_{\alpha} \cdot \underline{a}_{\alpha}$, one has

$$
(\underline{\underline{\Sigma}} \cdot \underline{n}) \cdot \underline{a}_{1}=(\underline{\underline{\sigma}} \cdot \underline{n}) \cdot \underline{a}_{1}=\sigma_{1 \alpha} n_{\alpha}=0 \quad \text { on } \Gamma \text { where } \sigma_{1 \alpha}=\mu\left(\mathrm{u}_{1, x_{\alpha}}+\mathrm{u}_{\alpha, x_{1}}\right)
$$

Since $\mathrm{u}_{1, x_{\alpha}}=O\left(\mathrm{u}_{1} / h\right)$ and $\mathrm{u}_{\alpha, x_{1}}=O\left(\mathrm{u}_{\alpha} / L\right)$, the vanishing of $\sigma_{1 \alpha}$ on $\Gamma$ requires

$$
O\left(\frac{\mathrm{u}_{1}}{h}\right)=O\left(\frac{\mathrm{u}_{\alpha}}{L}\right) \text { i.e. } O\left(\mathrm{u}_{1}\right)=\varepsilon O\left(\mathrm{u}_{\alpha}\right)
$$

Thus, the normal motions associated to transverse motions are of one order inferior. Consequently, the motions are expressed in the following rescaled form:

$$
\underline{\mathrm{u}}=\varepsilon u_{1} \underline{a}_{1}+u_{\alpha} \underline{a}_{\alpha} \quad \text { with } O\left(u_{1}\right)=O\left(u_{\alpha}\right)
$$

Considering motions on the form (21), the reduced strain tensors (16) are of different order:

$$
\mathrm{e}_{\mathrm{n}}=\varepsilon u_{1, x_{1}}, \quad \underline{\mathrm{e}}_{\mathrm{t}}=\left[\left(u_{1, y_{\alpha}}+u_{\alpha, x_{1}}\right) / 2\right] \underline{a}_{\alpha}, \quad \underline{\underline{\mathrm{e}}}_{\mathrm{s}}=\varepsilon^{-1}\left[\left(u_{\alpha, y_{\beta}}+u_{\beta, y_{\alpha}}\right) / 2\right]\left(\underline{a}_{\alpha} \otimes \underline{a}_{\beta}+\underline{a}_{\beta} \otimes \underline{a}_{\alpha}\right)
$$

and the effective stress tensor reads (where $\underline{\underline{I}}_{s}=e_{2} \otimes e_{2}+e_{3} \otimes e_{3}$ ):

$$
\sigma_{\mathrm{n}}=2 \mu \mathrm{e}_{\mathrm{n}}+\lambda\left(\operatorname{tr}\left(\underline{\underline{\mathrm{e}}}_{\mathrm{s}}\right)+\mathrm{e}_{\mathrm{n}}\right), \quad \underline{\sigma}_{\mathrm{t}}=2 \mu \underline{\mathrm{e}}_{\mathrm{t}}, \quad \underline{\underline{\sigma}}_{\mathrm{s}}=2 \mu \underline{\underline{\mathrm{e}}}_{\mathrm{s}}+\lambda\left(\operatorname{tr}\left(\underline{\underline{\mathrm{e}}}_{\mathrm{s}}\right)+\mathrm{e}_{\mathrm{n}}\right) \underline{\underline{\mathrm{I}}}_{\mathrm{s}}
$$

Consequently, $\underline{\sigma}_{\mathrm{t}}$ is of zero order while $\sigma_{\mathrm{n}}$ and $\underline{\sigma}_{\mathrm{s}}$ contain terms of order $\varepsilon^{-1}$ and $\varepsilon$. Finally, the effect of pressure $p$ is significant when its magnitude is of the order of the isotropic part of the elastic stress $\sigma_{\mathrm{n}}$ and $\underline{\underline{\sigma}}_{\mathrm{s}}$. 
Recall that, in this section, the current beam section is free of surface and volume forces. As for the physics in the poroelastic media, the following situation is addressed (other situations are discussed in Section 5):

- The compressibility of both fluid and solid are assumed to be of the same order: $K_{\mathrm{f}}=O\left(K_{b}\right)$.

- The fluid flow in the pores is dominated by the viscous effects. This means that $K=\mathcal{K}(1+O(\varepsilon)$ ), which implies a low frequency range, namely $\omega / \omega_{c}=O(\varepsilon)$, and vanishing inertial flow effect, i.e. $i \omega \rho_{\mathrm{f}} \mathrm{K} / \eta=O(\varepsilon)$. According to the asymptotic approach, and focusing on the leading order only, the $O(\varepsilon)$ inertia terms are disregarded.

- The fluid transfer trough permeability is balanced by both solid and fluid volume variations at the scale of the beam section. This means that:

$$
O\left(\frac{p}{M}\right)=\frac{\mathcal{K}}{i \omega \eta} O\left(\frac{p}{h^{2}}\right)=\varepsilon^{2} \frac{\mathcal{K}}{i \omega \eta} O\left(\Delta_{x}(p)\right)
$$

Hence the permeability has to be rescaled by a factor $\varepsilon^{2}$ and the balance equations (11)-(12) with the boundary conditions are re-expressed in the rescaled form as follows:

$$
\begin{aligned}
& \left(\Sigma_{i 1, x_{1}}+\varepsilon^{-1} \Sigma_{i \alpha, y_{\alpha}}\right) \underline{a}_{i}=\underline{0} \quad \text { in } S \\
& \underline{\underline{\Sigma}} \cdot \underline{n}=\underline{0} \quad \text { on } \Gamma \\
& \frac{\varepsilon^{2} \mathcal{K}}{i \omega \eta}\left(\varepsilon^{-2} \Delta_{y}(p)+p_{, x_{1} x_{1}}\right)-\frac{p}{M}-\alpha\left(\varepsilon^{-1} u_{\alpha, y_{\alpha}}+u_{1, x_{1}}\right)=0 \quad \text { in } S \\
& \frac{\xi}{h} \cdot p+\underline{\operatorname{grad}}(p) \cdot \underline{n}=0 \quad \text { on } \Gamma
\end{aligned}
$$

Conveniently, these equations are split into:

- The axial equilibrium of the section. It expresses the balance of the stress vector $\underline{\sigma}_{\mathrm{t}}$ under homogeneous boundary conditions; the axial gradient of the effective normal stress $\Sigma_{\mathrm{n}}=\sigma_{\mathrm{n}}-\alpha p$ acts as a forcing term:

$$
\begin{aligned}
& \Sigma_{\mathrm{n}, x_{1}}+\varepsilon^{-1} \operatorname{div}_{y}\left(\underline{\sigma}_{\mathrm{t}}\right)=0 \text { in } S \\
& \underline{\sigma}_{\mathrm{t}} \cdot \underline{n}=0 \text { on } \Gamma
\end{aligned}
$$

- The in-plane equilibrium of the section. It expresses, (i) the balance of the in-plane total stress tensor $\underline{\underline{\Sigma}}_{s}-$ the axial gradient of vector $\underline{\sigma}_{t}$ being a forcing term - under homogeneous boundary conditions and (ii) the fluid mass balance where $p_{, x_{1} x_{1}}$ and $u_{1, x_{1}}$ act as forcing terms - under leakage boundary conditions:

$$
\begin{aligned}
& \underline{\sigma}_{\mathrm{t}, x_{1}}+\varepsilon^{-1} \underline{\operatorname{div}}_{y}\left(\underline{\underline{\Sigma}}_{s}\right)=\underline{0} \text { in } S \\
& \underline{\underline{\Sigma}}_{\mathrm{s}} \cdot \underline{\underline{n}}=\underline{0} \quad \text { on } \Gamma \\
& {\left[\frac{\varepsilon^{2} \mathcal{K}}{i \omega \eta} p_{, x_{1}}-\alpha u_{1}\right]_{, x_{1}}+\operatorname{div}_{y}\left[\frac{\mathcal{K}}{i \omega \eta} \underline{\operatorname{grad}}_{y}(p)\right]-\varepsilon^{-1} \alpha \operatorname{div}_{y}\left(u_{\alpha} \underline{a}_{\alpha}\right)-p / M=0 \quad \text { in } S} \\
& \frac{\xi}{h} \cdot p+\underline{\operatorname{grad}}(p) \cdot \underline{n}=0 \quad \text { on } \Gamma
\end{aligned}
$$

\section{Derivation of beam behavior by asymptotic expansions}

The aim is to determine the behavior of slender bodies, i.e. attained at small $\varepsilon$. In this view, we seek for the variables in the form of expansions in power of $\varepsilon$. These latter must respect the beam kinematics (21), i.e. $\underline{u}=\varepsilon u_{1} \underline{a}_{1}+u_{\alpha} \underline{a}_{\alpha}$. As a consequence, a condensed form of the expansions can be specified in advance. Indeed, using the reduced strain tensors (22) to express the reduced stress tensors (23) and inserting them into the balance and boundary equations (24)-(29) yield to scaled problems expressed in function of $u_{1}, u_{\alpha}$ and $p$ (of the same order than $\sigma_{n}$ ). The balance equations and boundary condition contain either terms in odd power of $\varepsilon$ (axial balance, mass balance, in-plane boundary condition) or terms in even power of $\varepsilon$ (in-plane balance, axial boundary condition). Since the terms of the equations "jump" from a factor $\varepsilon^{2}$, it is sufficient to expand $u_{i}$ in even powers of $\varepsilon$ and $p$ in odd power of $\varepsilon$. Thus, the appropriate expansion reads

$$
\underline{u}=\sum_{i=0} \varepsilon^{2 i}\left[u_{\alpha}^{2 i} \underline{a}_{\alpha}+\varepsilon\left(u_{1}^{2 i+1} \underline{a}_{1}\right)\right], \quad u_{\alpha}=\sum_{i=0} \varepsilon^{2 i} u_{\alpha}^{2 i}, \quad u_{1}=\sum_{i=0} \varepsilon^{2 i} u_{1}^{2 i+1}, \quad p=\varepsilon \sum_{i=-1} \varepsilon^{2 i} p^{2 i+1}
$$


Consequently, the axial $(\mathrm{n})$ and in plane $(\mathrm{s})$ - respectively out of plane $(\mathrm{t})$ - reduced strain and stress tensors $(22),(23)$ are expanded in odd - respectively even - powers of $\varepsilon$ :

$$
\begin{aligned}
& \underline{\underline{\mathrm{e}}}_{\mathrm{s}}=\varepsilon \sum_{i=-1} \varepsilon^{2 i} \underline{\underline{\mathrm{e}}}_{\mathrm{s}}^{2 i}, \quad \underline{\mathrm{e}}_{\mathrm{t}}=\sum_{i=0} \varepsilon^{2 i} \underline{\mathrm{e}}_{\mathrm{t}}^{2 i}, \quad \mathrm{e}_{\mathrm{n}}=\varepsilon \sum_{i=0} \varepsilon^{2 i} \mathrm{e}_{\mathrm{n}}^{2 i+1} \\
& \underline{\underline{\sigma}}_{\mathrm{s}}=\varepsilon \sum_{i=-1} \varepsilon^{2 i} \underline{\underline{\sigma}}_{\mathrm{s}}^{2 i}, \quad \underline{\sigma}_{\mathrm{t}}=\sum_{i=0} \varepsilon^{2 i} \underline{\sigma}_{\mathrm{t}}^{2 i}, \quad \sigma_{\mathrm{n}}=\varepsilon \sum_{i=-1} \varepsilon^{2 i} \sigma_{\mathrm{n}}^{2 i+1}
\end{aligned}
$$

Note that this type of expansions is usual when dealing with slender structures, see for instance Trabucho and Viano (1996) and Boutin and Soubestre (2011).

\subsection{Asymptotic solution}

The asymptotic solution is derived by introducing expansions (30) in (24)-(29). Separating the terms of different order leads to a series of problems to be solved successively. The comprehensive resolution require five steps developed here. The two first steps demonstrate the validity of the Euler-Bernoulli kinematic for poroelastic beams. The third step provides the stress-strain state in the section, and necessitates the resolution of in-plane pressure diffusion problems. The poroelastic constitutive laws and the balance equations at the leading order are derived from the two next steps.

\subsubsection{The first problem: uniform section translation}

Eqs. $\left(26_{\varepsilon^{-2}}\right),\left(27_{\varepsilon^{-1}}\right),\left(28_{\varepsilon^{-2}}\right)$, and $\left(29_{\varepsilon^{-1}}\right)$ govern the in-plane motion $\underline{u}^{0}=u_{\alpha}^{0} \underline{a}_{\alpha}$ and the pressure $p^{-1}$.

$$
\begin{aligned}
& \operatorname{div}_{y}\left(\underline{\underline{\Sigma}}_{\mathrm{s}}^{-1}\right)=\underline{0} \text { in } S \\
& \underline{\underline{\Sigma}}_{\mathrm{s}}^{-1} \cdot \underline{\underline{n}}=\underline{0} \quad \text { on } \Gamma \\
& \operatorname{div}_{y}\left(\frac{\mathcal{K}}{i \omega \eta} \underline{\operatorname{grad}} \underline{y}_{y}\left(p^{-1}\right)\right)-\frac{p^{-1}}{M}=\underline{0} \quad \text { in } S \\
& \frac{\xi}{h} \cdot p^{-1}+\underline{\operatorname{grad}}\left(p^{-1}\right) \cdot \underline{n}=0 \quad \text { on } \Gamma
\end{aligned}
$$

This is a problem of plane poroelasticity without any loading (neither in $S$ nor on $\Gamma$ ). Therefore, $\underline{u}^{0}$ is a rigid motion of the section in its plane, i.e. a translation $\underline{U}^{0}$ and a rotation $\Omega^{-1} \underline{a}_{1}$, and the pressure vanishes:

$$
\underline{u}^{0}=u_{\alpha}^{0} \underline{a}_{\alpha}, \quad u_{\alpha}^{0}=U_{\alpha}^{0}\left(x_{1}\right)+\Omega^{-1}\left[\underline{a}_{1} \times \underline{y}\right]_{\alpha}, \quad p^{-1}=0
$$

Moreover, $\underline{\underline{e}}_{s y}\left(\underline{u}^{0}\right)=\underline{\underline{0}}$, then ${\underline{\underline{\sigma_{s}}}}^{-1}=\underline{\underline{0}}$ and $\sigma_{\mathrm{n}}^{-1}=\lambda \operatorname{div}_{y}\left(\underline{u}^{0}\right)=0$. Thus:

$$
\underline{\underline{\mathrm{e}}}^{-1}=\underline{\underline{0}}, \quad \underline{\underline{\Sigma}}^{-1}=\underline{\underline{\sigma}}^{-1}=\underline{\underline{0}}
$$

The translation $\underline{U^{0}}$ and the rotation $\Omega^{-1}$ (of order ${ }^{-1}$ to respect the scaling of the zero order displacement $\Omega^{-1} h=O(1)$ ) are two independent kinematics that may be treated separately. They arise at the same order because the assumption of zero order transverse motion does not distinguish translation and rotation. Nevertheless, their relative order of magnitude may differ physically. Without restricting the generality of the further developments, we will consider that the rotation is of lesser order than the translation, i.e. $\Omega^{-1}=0$, and leave the treatment of the section rotation for superior orders.

\subsubsection{The second problem: Euler-Bernoulli kinematics}

Eqs. $\left(24_{\varepsilon^{-1}}\right)$ and $\left(25_{\varepsilon^{0}}\right)$ deal with the axial motion $u_{1}^{1}$ and the axial balance of $\underline{\sigma}_{t}^{0}$. Taking into account the fact that $\Sigma_{\mathrm{n}}^{-1}=0$, we have

$$
\begin{aligned}
& \operatorname{div}_{y}\left(\underline{\sigma}_{\mathrm{t}}^{0}\right)=0 \text { in } S, \quad \underline{\sigma}_{\mathrm{t}}^{0}=\mu\left(u_{1, y_{\alpha}}^{1}+U_{\alpha, x_{1}}^{0}\right) \underline{a}_{\alpha} \\
& \underline{\sigma}_{\mathrm{t}}^{0} \cdot \underline{n}=0 \text { on } \Gamma
\end{aligned}
$$

This is a shear elastic problem, with $U_{\alpha, x_{1}}^{0}$ as forcing term and with free boundary. It admits the following solution:

$$
\begin{aligned}
& \underline{u}^{1}=u_{1}^{1} \underline{a}_{1} \quad \text { with } u_{1}^{1}=-\underline{y} \cdot \underline{U}_{, x_{1}}^{0}+U_{1}^{1}\left(x_{1}\right) \\
& \underline{\mathrm{e}}_{\mathrm{t}}^{0}=0, \quad \underline{\sigma}_{\mathrm{t}}^{0}=0, \quad \text { hence } \underline{\underline{\mathrm{e}}}^{0}=0, \quad \underline{\underline{\Sigma}}^{0}=\underline{\underline{\sigma}}^{0}=0
\end{aligned}
$$

This shows that at the leading order, the out of plane motion of the section consists in the usual kinematics of the EulerBernoulli beam. Despite the fact that the relative magnitude of (i) the rigid out of plane rotation (of vector $\underline{U}_{, x_{1}}^{0} \times \underline{a}_{1}$ ) and (ii) the uniform vertical translation $U_{1}^{1} \underline{a}_{1}$ may physically differ, it is convenient to treat them conjointly. 
4.1.3. The third problem: poroelastic stress-strain state

Eqs. $\left(24_{\varepsilon^{0}}\right),\left(27_{\varepsilon}\right),\left(28_{\varepsilon^{0}}\right)$, and $\left(29_{\varepsilon}\right)$ concern the in-plane field $\underline{u}^{2}$ and the pressure $p^{1}$. Using the previous results, the problem takes the form herebelow. Note that the forces and volume balances undergo the same forcing term $u_{1, x_{1}}^{1}$ which cumulates the bending forcing $-\underline{y} \cdot U_{, x_{1} x_{1}}^{0}$ and the compression forcing $U_{1, x_{1}}^{1}$ :

$$
\begin{aligned}
& \underline{\underline{\Sigma}}_{\mathrm{s}}^{1}=2 \mu \underline{\underline{\mathrm{e}}}_{\mathrm{s} y}\left(\underline{u}^{2}\right)+\lambda\left[\operatorname{div}_{y}\left(\underline{u}^{2}\right)+u_{1, x_{1}}^{1} \underline{\underline{I}}_{\mathrm{s}}-\alpha p^{1} \underline{\underline{I}}_{\mathrm{s}}, \quad \underline{\operatorname{div}}_{y}\left(\underline{\underline{\Sigma}}_{\mathrm{s}}^{1}\right)=\underline{0} \text { in } S\right. \\
& \underline{\underline{\Sigma}}_{\mathrm{s}}^{1} \cdot \underline{n}=\underline{0} \quad \text { on } \Gamma \\
& \operatorname{div}_{y}\left(\frac{\mathcal{K}}{i \omega \eta} \underline{\operatorname{grad}}_{y}\left(p^{1}\right)\right)-\frac{p^{1}}{M}-\alpha\left[\operatorname{div}_{y}\left(\underline{u}^{2}\right)+u_{1, x_{1}}^{1}\right]=0 \quad \text { in } S \\
& \frac{\xi}{h} \cdot p+\underline{\operatorname{grad}}(p) \cdot \underline{n}=0 \text { on } \Gamma
\end{aligned}
$$

The solution $\left(\underline{u}^{2}, p^{1}\right)$ of this plane poroelastic problem is decomposed into the elastic solution $\left(\underline{u}^{e}\right)$ that prevails when the material is purely elastic (drained state) and a poroelastic contribution $\left(\underline{\widehat{u}}^{2}, p^{1}\right)$, i.e. $\left(\underline{u}^{2}, p^{1}\right)=\left(\underline{u}^{e}, 0\right)+\left(\underline{\widehat{u}}^{2}, p^{1}\right)$. The details of the resolution are given in Appendix. The poroelastic contribution involves specific solutions, denoted ( $\underline{c}$, $\zeta$ ) for compression, and $\left(\underline{b}^{\alpha}, \psi^{\alpha}\right)$ for bending in direction $\underline{a}_{\alpha}$. The complete solution yields to the following strain, effective and total stress tensors at the first order:

$$
\begin{aligned}
& \left.\underline{\underline{\mathrm{e}}}^{1}=\left[\underline{a}_{1} \otimes \underline{a}_{1}-v \underline{\underline{I}}_{\mathrm{s}}\right]\left[-y_{\alpha} \cdot U_{\alpha, x_{1} x_{1}}^{0}+U_{1, x_{1} x_{1}}^{1}\right]+(1-2 v) \alpha \frac{A}{\lambda+\mu} \underline{\underline{\mathrm{e}}}_{s}\left(\underline{b}^{\alpha}\right) U_{\alpha, x_{1} x_{1}}^{0}+\underline{\underline{\mathrm{e}}}_{\mathrm{s}}(\underline{c}) U_{1, x_{1}}^{1}\right] \\
& \left.\underline{\underline{\sigma}}^{1}=E\left[-y_{\alpha} \cdot U_{\alpha, x_{1} x_{1}}^{0}+U_{1, x_{1} x_{1}}^{1}\right] \underline{a}_{1} \otimes \underline{a}_{1}+(1-2 v) \alpha \frac{A}{\lambda+\mu}\left\{\lambda\left\{\operatorname{div}_{y}\left(\underline{b}^{\alpha}\right) U_{\alpha, x_{1} x_{1}}^{0}+\operatorname{div}_{y}(\underline{c}) U_{1, x_{1}}^{1}\right)\right\} \underline{\underline{I}}+2 \mu\left\{\underline{\underline{\mathrm{e}}}_{s}\left(\underline{b}^{\alpha}\right) U_{\alpha, x_{1} x_{1}}^{0}+\underline{\underline{\mathrm{e}}}_{s}(\underline{c}) U_{1, x_{1}}^{1}\right\}\right\} \\
& \underline{\underline{\Sigma}}^{1}=\underline{\underline{\sigma}}^{1}-(1-2 v) \alpha^{2} A\left[\psi^{\alpha} U_{\alpha, x_{1} x_{1}}^{0}+\zeta U_{1, x_{1}}^{1}\right] \underline{\mathrm{I}}
\end{aligned}
$$

The poroelastic strain and effective stress states at the leading order do not follow the form that prevails in elastic beams. Nevertheless, using identities (62), the mean total stress tensor reduces to an axial component as in elastic beams:

$$
\int_{S} \underline{\underline{\Sigma}}^{1} d s=\left[\underline{a}_{1} \otimes \underline{a}_{1}\right] \int_{S} \Sigma_{n}^{1} d s ; \quad \int_{S} y_{\alpha} \underline{\underline{\Sigma}}^{1} d s=\left[\underline{a}_{1} \otimes \underline{a}_{1}\right] \int_{S} y_{\alpha} \Sigma_{n}^{1} d s
$$

and from (74)-(75), we have:

$$
\begin{aligned}
& \int_{S} \Sigma_{n}^{1} d s=E S^{\prime} U_{1, x_{1}}^{1}-[(1-2 v) \alpha]^{2} A \int_{S}\left[\psi^{\alpha} U_{\alpha, x_{1} x_{1}}^{0}+\zeta U_{1, x_{1}}^{1}\right] d s \\
& \int_{S} y_{\alpha} \Sigma_{n}^{1} d s=-E I_{\alpha}^{\prime} U_{\alpha, x_{1} x_{1}}^{0}-[(1-2 v) \alpha]^{2} A \int_{S} y_{\alpha}\left[\psi^{\alpha} U_{\alpha, x_{1} x_{1}}^{0}+\zeta U_{1, x_{1}}^{1}\right] d s
\end{aligned}
$$

Note also that, conversely to the 3D-poroelasticity where the pressure is an independent variable, the pressure in poroelastic beams is directly related to the solid deformation and becomes a hidden variable. This also applies to the pressure gradient, and in turn to the Darcy's velocity, whose leading order components (the axial is of one order smaller than the in-plane) read

$$
\begin{aligned}
& \phi i \omega\left(\underline{u}_{\mathrm{f}}-\underline{u}\right)_{\beta}=-(1-2 v) \alpha\left[\psi_{, y_{\beta}}^{\alpha} U_{\alpha, x_{1} x_{1}}^{0}+\zeta_{, y_{\beta}} U_{1, x_{1}}^{1}\right] \frac{A \mathcal{K}}{\eta} \\
& \phi i \omega\left(\underline{u}_{\mathrm{f}}-\underline{u}\right)_{1}=-(1-2 v) \alpha\left[\psi^{\alpha} U_{\alpha, x_{1} x_{1} x_{1}}^{0}+\zeta U_{1, x_{1} x_{1}}^{1}\right] \frac{A \mathcal{K}}{\eta}
\end{aligned}
$$

Then, from the order of magnitude of the terms, we deduce that

$$
\left(\underline{u}_{\mathrm{f}}-\underline{u}\right)_{i}=\varepsilon^{2} \mathrm{O}\left(U_{i}\right)
$$

\subsubsection{The fourth problem: axial and momentum balances}

Eqs. $\left(24_{\varepsilon}\right)$ and $\left(25_{\varepsilon^{2}}\right)$ concern the axial balance of $\underline{\sigma}_{\mathrm{t}}^{2}$ where $\Sigma_{\mathrm{n}, x_{1}}^{1}$ acts as a source term:

$$
\begin{aligned}
& \sum_{\mathrm{n}, x_{1}}^{1}+\operatorname{div}_{y}\left(\underline{\sigma}_{\mathrm{t}}^{2}\right)=0 \text { in } S, \quad \underline{\sigma}_{\mathrm{t}}^{2}=\mu\left[u_{1, y_{\alpha}}^{3}+u_{\alpha, x_{1}}^{2}\right] \underline{a}_{\alpha} \\
& \underline{\sigma}_{\mathrm{t}}^{2} \cdot \underline{n}=0 \quad \text { on } \Gamma
\end{aligned}
$$

The global axial and momentum balance equations of the beam section are established as described in Section 2.4 . Noticing that the $y$-integral over the section of a quantity of order $i$ multiplied by $y_{\alpha}^{j}$ is of order $i+(2+j)$, one obtains (in absence of body and surface forces):

$$
N_{, x_{1}}^{3}=0, \quad N^{3}=\int_{S} \sum_{\mathrm{n}}^{1} d s
$$




$$
\underline{M}_{, x_{1}}^{4}-\underline{T}^{4}=\underline{0}, \quad M_{\alpha}^{4}=\int_{S} \Sigma_{\mathrm{n}}^{1} y_{\alpha} d s
$$

The beam behavioral laws relating the normal force and the transverse momentum to the longitudinal strain and curvature are deduced from the expression (32) of $\underline{\underline{\Sigma}}_{n}^{1}$.

In elastic beams, the compression and bending $\bar{m}$ mechanisms are uncoupled when expressed in the main $y$-frame. In general, this is no longer true for poroelastic beams, because the $y$-frame that enables uncoupling depends on the pressure distribution (hence on the frequency) and does not necessarily coincide with the main $y$-frame. However, if the section is bi-symmetric, the pressure fields respect the bi-symmetry, and in this case (see (70)):

$$
\int_{S} \psi^{\alpha} d s=\int_{S} y_{\alpha} \zeta d s=0
$$

Thus, the compression and bending mechanisms are uncoupled in the main $y$-frame. Then, using the identities (74)-(75), the beam constitutive laws expressed in the symmetry axis of the bi-symmetric section simply read

$$
\begin{aligned}
& N^{3}=\left[E\left|S^{\prime}\right|-[(1-2 v) \alpha]^{2} A \int_{S} \zeta d s\right] U_{1, x_{1}}^{1} \\
& M_{\alpha}^{4}=-\left[E I_{\alpha}^{\prime}+[(1-2 v) \alpha]^{2} A \int_{S} y_{\alpha} \psi^{\alpha} d s\right] U_{\alpha, x_{1} x_{1}}^{0}
\end{aligned}
$$

In addition, the mean and "moment" of the axial Darcy flux are also uncoupled since from (33) and (37):

$$
\begin{aligned}
& \phi i \omega \int_{S}\left(\underline{u}_{\mathrm{f}}-\underline{u}\right)_{1} d s=-(1-2 v) \alpha \frac{A \mathcal{K}}{\eta}\left[\int_{S} \zeta d s\right] U_{1, x_{1} x_{1}}^{1} \\
& \phi i \omega \int_{S} y_{\alpha}\left(\underline{u}_{\mathrm{f}}-\underline{u}\right)_{1} d s=-(1-2 v) \alpha \frac{A \mathcal{K}}{\eta}\left[\int_{S} y_{\alpha} \psi^{\alpha} d s\right] U_{\alpha, x_{1} x_{1} x_{1}}^{0}
\end{aligned}
$$

The derivation of $u_{1}^{3}$ and $\underline{\sigma}_{t}^{2}$, not necessary at this stage, is given in Appendix.

\subsubsection{The fifth problem: transverse and torsion balances}

Eqs. $\left(26_{\varepsilon^{2}}\right)$ and $\left(27_{\varepsilon^{3}}\right)$ express the in-plane balance of $\underline{\underline{\sigma}}_{s}^{3}$ in presence of the forcing term $\underline{\sigma}_{t, x_{1}}^{2}$.

$$
\begin{aligned}
& \underline{\sigma}_{t, x_{1}}^{2}+\underline{\operatorname{div}}_{y}\left(\underline{\underline{\sigma}}_{s}^{3}\right)=\underline{0} \text { in } S \\
& \underline{\underline{\sigma}}_{\mathrm{s}}^{3} \cdot \underline{n}=\underline{0} \quad \text { on } \Gamma
\end{aligned}
$$

Following Section 2.4, two balance equations are deduced (without body and surface forces):

$$
\begin{aligned}
& \underline{T}_{, x_{1}}^{4}=0, \quad T_{\alpha}^{4}=\int_{S} \underline{\sigma}_{\mathrm{t}}^{2} \cdot \underline{a}_{\alpha} d s \\
& M_{1, x_{1}}^{5}=0, \quad M_{1}^{5}=\int_{S}\left[\underline{y} \times \underline{\sigma}_{\mathrm{t}}^{2}\right] \cdot \underline{a}_{1} d s=\mu I_{t}^{\prime} \Omega_{, x_{1}}^{1}
\end{aligned}
$$

The torsion law relating $M_{1}^{5}$ to $\Omega_{, x_{1}}^{1}$ valid for bi-symmetric sections is proved in Appendix $\left(I_{t}^{\prime}\right.$ is the torsion inertia that accounts for warping). Notice that, the bi-symmetric poroelastic torsion law coincides with that of purely elastic beams. Conversely, non bi-symmetric sections may introduce torsion-bending-compression coupling via the poroelastic effects.

To sum up, the leading order description of poroelastic beams with current section free of loading is given by the set (35), (36), (38)-(41).

\subsection{Complete beam description}

This section provides the description of poroelastic beams in presence of body and/or contact forces on the current section. The quasi-static and dynamic descriptions are established in harmonic regime, then expressed in time domain more convenient for transient loading. For simplicity, we focus on bi-symmetric sections.

\subsubsection{Loaded poroelastic beam-quasi-static harmonic regime}

Let us examine body forces $\underline{f}=f_{i} \underline{a}_{i}$ - such that $\underline{\operatorname{div}}(\underline{\underline{\Sigma}})=f$ - and contact forces $\underline{g}=g_{i} \underline{a}_{i}$ - such that $\underline{g}=\underline{\underline{\Sigma}} \cdot \underline{n}$ on $\Gamma$ - that can be applied on the current section while being compatible with the above derived beam description. First, they should not break the axial/transverse scale separation so that they may be expressed as $f\left(x_{1}, y_{\alpha}\right), g\left(x_{1}, y_{\alpha}\right)$. Second, they should be small enough not to disturb the leading kinematics of the section. This is the case when $\underline{f}$ and $\underline{g}$ are of the orders:

$$
f_{1}=\varepsilon f_{1}^{1}, g_{1}=\varepsilon^{2} g_{1}^{2} \quad ; \quad f_{\alpha}=\varepsilon^{2} f_{\alpha}^{2}, g_{\alpha}=\varepsilon^{3} g_{\alpha}^{3}
$$


Indeed, in that case, the problems remain identical up to the fourth one. Only the global equilibrium is modified by $f$ and $g$ whose averaged values on $S$ and $\Gamma$ act as sources. Hence, the balance become:

$$
\begin{aligned}
& N_{x_{1}}^{3}=\int_{S} f_{1}^{1} d s+\int_{\Gamma} g_{1}^{2} d \gamma \\
& M_{\alpha, x_{1}}^{4}-T_{\alpha}^{4}=\int_{S} y_{\alpha} f_{1}^{1} d s+\int_{\Gamma} y_{\alpha} g_{1}^{2} d \gamma, \quad T_{\alpha, x_{1}}^{4}=\int_{S} f_{\alpha}^{2} d s+\int_{\Gamma} g_{\alpha}^{3} d \gamma \\
& M_{1, x_{1}}^{5}=\underline{a}_{1} \cdot \int_{S} \underline{y} \times \underline{f}^{2} d s+\underline{a}_{1} \cdot \int_{\Gamma} \underline{y} \times \underline{g}^{3} d \gamma
\end{aligned}
$$

Note that, in the fourth problem, $u_{1}^{3}$ is modified by $f_{1}^{1}$ and $g_{1}^{2}$. Thus the uncoupling of bending and torsion requires that $f_{1}^{1}$ and $g_{1}^{2}$ respect the bi-symmetry of the section.

Smaller magnitudes of $f$ and $g$ lead to the unloaded beam description (at the leading order). Conversely, larger amplitudes of $f$ or $g$ are incompatible with a beam model: the specific Euler-Bernoulli beam kinematics intrinsically related to the strain-stress states of compression and bending would be lost. In other words, a 3D kinematics and 3D strain-stress states at the leading order would be necessary to balance such large amplitude loading.

For practical applications, it is more convenient to express the description in the usual unscaled form. This is obtained by coming back to the unscaled variables $x_{i}$ with the inverse change of variable $x_{\alpha}=\varepsilon y_{\alpha}$, by considering the physically observable quantities $\varepsilon^{i} Q^{i}$ instead of the scaled quantities $Q^{i}$, and by expressing the parameters in the system $x_{i}$ (i.e. practically, with the same units in the section and in the beam axis). Furthermore, there is no constraint on the relative order of magnitude of the uncoupled, then independent, mechanisms. For this reason, the exponent specifying the order may be dropped (for the leading order description). Hence, denoting the unscaled global loading by

$$
\begin{aligned}
& \underline{F}=\int_{S} \underline{f} d S \quad ; \quad \underline{G}=\int_{\Gamma} \underline{g} d \Gamma \\
& \underline{C}_{\alpha}=\int_{S} x_{\alpha} f_{1} d s, \quad C_{1}=\underline{a}_{1} \cdot \int_{S} \underline{x} \times \underline{f} d s \quad ; \quad D_{\alpha}=\int_{\Gamma} x_{\alpha} f_{1} d \Gamma, D_{1}=\underline{a}_{1} \cdot \int_{\Gamma} \underline{x} \times \underline{f} d \Gamma
\end{aligned}
$$

the description of the loaded poroelastic beam with the usual variables reads

\section{- Kinematics}

$$
U=\left(U_{1}+x_{\alpha} \frac{\partial U_{\alpha}}{\partial x_{1}}\right) \underline{a}_{1}+U_{\alpha} \underline{a}_{\alpha}+\Omega \underline{a}_{1} \times\left(x_{\alpha} \underline{a}_{\alpha}\right)
$$

- Normal force $N$ and mean vertical motion $U_{1}$

$$
\begin{aligned}
& \frac{\partial N}{\partial x_{1}}=F_{1}+G_{1} \\
& N=\widehat{E S} \frac{\partial U_{1}}{\partial x_{1}} \quad ; \quad \widehat{E S}=E|S|-[(1-2 v) \alpha]^{2} A \int_{S} \zeta d S
\end{aligned}
$$

- Transverse forces $T_{\alpha}$, momentum $M_{\alpha}$ and mean transverse motion $U_{\alpha}$

$$
\begin{aligned}
& \frac{\partial M_{\alpha}}{\partial x_{1}}-T_{\alpha}=C_{\alpha}+D_{\alpha} \\
& \frac{\partial T_{\alpha}}{\partial x_{1}}=0 \\
& M_{\alpha}=-\widehat{E I_{\alpha}} \frac{\partial^{2} U_{\alpha}}{\partial x_{1}^{2}} ; \quad \widehat{E I_{\alpha}}=E I_{\alpha}+[(1-2 v) \alpha]^{2} A \int_{S} x_{\alpha} \psi^{\alpha} d S
\end{aligned}
$$

- Torsion momentum $M_{1}$ and in-plane rotation (torsion) $\Omega$

$$
\frac{\partial M_{1}}{\partial x_{1}}=C_{1}+D_{1} \quad ; \quad M_{1}=\mu I_{t} \frac{\partial \Omega}{\partial x_{1}}
$$

At the extremities of the poroelastic beam, the boundary conditions are of the same nature as in elastic beams (i.e. forces and moments and/or displacement rotations) according to the St. Venant principle. As the pressure is a hidden variable and the fluid flow is of one order less than solid motions, the matching of specified conditions for pressure or fluid flow can only be realized through correctors introducing boundary layers, following a similar matching approach as developed by Panasenko (2000) and Buannic and Cartraud (2001) for elastic beams. 


\subsubsection{Dynamics of poroelastic beam}

The dynamics of beams unloaded on their surface $(g=0)$ is treated in a similar way, considering harmonic regimes at a frequency sufficiently low, $\omega / \omega_{c} \leq O(\varepsilon)$, to insure vanishing inertia effect in the flow, i.e. $i \omega \rho_{\mathrm{f}} \mathrm{K} / \eta=O(\varepsilon)$ (thus the mass balance considered in Section 4.1.3 remains valid). The inertial body force $f=-\omega^{2}\left[(1-\phi) \rho_{\mathrm{s}} \underline{u}+\phi \rho_{\mathrm{f}} \underline{u}_{\mathrm{f}}\right]$ can be assessed provided that the inner state of the beam, previously determined, is respected. In that case, as established in (34), the relative fluid solid displacement is two order smaller than the solid displacement. Thus, at the leading order, the section displacement induces an inertial body force $-\omega^{2} \rho_{\mathrm{m}} \underline{u}$ that reads

$$
\underline{f}=-\omega^{2} \rho_{m}\left[\left(U_{1}^{1}+y_{\alpha} U_{\alpha, x_{1}}^{0}\right) \underline{a}_{1}+U_{\alpha}^{0} \underline{a}_{\alpha}+\Omega^{1} \underline{a}_{1} \times \underline{y}\right]
$$

This estimate only applies if $f$ does not interfere with beam behavior laws. As discussed for static loading, this requires that

$$
f_{1}=\varepsilon f_{1}^{1} \text { and } f_{\alpha}=\varepsilon^{2} f_{\alpha}^{2}
$$

For pure compression ( $U=U_{1}^{1} \underline{a}_{1}$ hence $\left.f=f_{1} \underline{a}_{1}\right)$ or pure torsion motions $\left(U=\Omega^{1} \underline{a}_{1} \times y\right.$ hence $\left.f=f_{\alpha} \underline{a}_{\alpha}\right)$ these conditions are satisfied independently. Only the global equilibrium is modified by the inertial terms whose averaged values on $S$ and $\Gamma$ act as sources. Hence, (35)-(41) become

$$
N_{, x_{1}}^{3}=-\rho_{m} \omega^{2}\left|S^{\prime}\right| U_{1}^{1}, \quad M_{1, x_{1}}^{5}=-\rho_{m} \omega^{2} I_{t}^{\prime} \Omega^{1}
$$

Note that in both cases the frequency is of the order of $\omega_{0}=O\left(\sqrt{E / \rho_{m}}(1 / L)\right)$.

As for transverse bending motions $\left(U_{\alpha}^{0} \underline{a}_{\alpha}+y_{\alpha} U_{\alpha, x_{1}}^{0} \underline{a}_{1}\right)$, inertial forces arise along both axial and in-plane axis and

$$
O\left(f_{1} / f_{\alpha}\right)=O\left(\left|y_{\alpha} U_{\alpha, x_{1}}^{0}\right| /\left|U_{\alpha}^{0}\right|\right)=O(h / L)=\varepsilon
$$

Then, the order of magnitude compatible with the beam description is

$$
f_{\alpha}=\varepsilon^{2} f_{\alpha}^{2} \text { and therefore } f_{1}=\varepsilon^{3} f_{1}^{3}
$$

Consequently, $\int_{S} f_{\alpha} d s=O\left(\varepsilon^{4}\right)$ and the mean translation inertia acts as a source in the global equilibrium of shear forces. As this estimate requires a frequency of the order of $O\left(\varepsilon \omega_{0}\right)=O\left(\sqrt{E / \rho_{m}}(1 / L)(h / L)\right)$, the frequency is rescaled as $\varepsilon \omega$. Moreover, since $\int_{S} y_{\alpha} f_{1} d s=O\left(\varepsilon^{6}\right)$, the out of plane rotation inertia term is negligible at the leading order of the bending moment balance (order 4). Finally, the balance equations (36)-(40) now read

$$
M_{\alpha, x_{1}}^{4}-T_{\alpha}^{4}=0, \quad T_{\alpha, x_{1}}^{4}=-\rho_{m}(\varepsilon \omega)^{2} S^{\prime} U_{\alpha}^{0}
$$

The restrictions on the magnitude of inertia terms express the fact that the characteristic size of the mode wave length must be larger than the size of the section. This imposes for compression and torsion modes a frequency domain such that $\omega=O\left(\omega_{0}\right)$; while for first bending mode the frequency domain is more restricted: $\omega<O\left(\omega_{0}\right) h / L$. Converse situations lead to lose the axialtransverse scale separation, hence the 1D beam description, and the poroelastic rod has to be described as a guide of waves.

Finally, rewritten with the usual variables, the poroelastic beam dynamics is described by the following set:

\section{- Kinematics}

$$
U=\left(U_{1}+x_{\alpha} \frac{\partial U_{\alpha}}{\partial x_{1}}\right) \underline{a}_{1}+U_{\alpha} \underline{a}_{\alpha}+\Omega \underline{a}_{1} \times\left(x_{\alpha} \underline{a}_{\alpha}\right)
$$

- Normal force $N$ and mean vertical motion $U_{1}$

$$
\begin{aligned}
& \frac{\partial N}{\partial x_{1}}=-\rho_{m} S \omega^{2} U_{1} \\
& N=\widehat{E S} \frac{\partial U_{1}}{\partial x_{1}} \quad ; \quad \widehat{E S}=E|S|-[(1-2 v) \alpha]^{2} A \int_{S} \zeta d S
\end{aligned}
$$

- Transverse forces $T_{\alpha}$, momentum $M_{\alpha}$ and mean transverse motion $U_{\alpha}$

$$
\begin{aligned}
& \frac{\partial M_{\alpha}}{\partial x_{1}}-T_{\alpha}=0 \\
& \frac{\partial T_{\alpha}}{\partial x_{1}}=-\rho_{m} S \omega^{2} U_{\alpha} \\
& M_{\alpha}=-\widehat{E I_{\alpha}} \frac{\partial^{2} U_{\alpha}}{\partial x_{1}^{2}} ; \quad \widehat{E I_{\alpha}}=E I_{\alpha}+[(1-2 v) \alpha]^{2} A \int_{S} x_{\alpha} \psi^{\alpha} d S
\end{aligned}
$$

- Torsion momentum $M_{1}$ and in-plane rotation (torsion) $\Omega$

$$
\frac{\partial M_{1}}{\partial x_{1}}=-\rho_{m} I_{t} \omega^{2} \Omega \quad ; \quad M_{1}=\mu I_{t} \frac{\partial \Omega}{\partial x_{1}}
$$




\subsubsection{Transient loading}

The difference between elastic and poroelastic beams lies in the behavior laws for normal force and momentum (38)-(39). The poroelastic compression stiffness $\widehat{E S}$ and bending inertia $\widehat{E I_{\alpha}}$ involve complex frequency dependant terms related to the pore pressure. When expressed in the time domain, the compression and bending laws involve convolution products, the kernel of which being the inverse Fourier transform of the terms related to pressure:

$$
\begin{aligned}
& N(t)=E|S| \frac{\partial U_{1}}{\partial x_{1}}-[(1-2 v) \alpha]^{2} A Z * \frac{\partial U_{1}}{\partial x_{1}} \quad ; \quad Z(t)=\int_{-\infty}^{\infty} \exp ^{-i \omega t}\left[\int_{S} \zeta(\omega) d S\right] d \omega \\
& M_{\alpha}(t)=-E I_{\alpha} \frac{\partial^{2} U_{\alpha}}{\partial x_{1}^{2}}+[(1-2 v) \alpha]^{2} A \Psi * \frac{\partial^{2} U_{\alpha}}{\partial x_{1}^{2}} \quad ; \quad \Psi(t)=\int_{-\infty}^{\infty} \exp ^{-i \omega t}\left[\int_{S} x_{\alpha} \psi^{\alpha}(\omega) d S\right] d \omega
\end{aligned}
$$

This induces damping of oscillation, relaxation and creep phenomena absent in elastic beams but usual in viscoelastic beams. However, conversely to these latter, the inelastic properties of poroelastic beams differ under compression or under bending deformations and vanish under torsion (for bi-symmetric sections).

Relaxation functions are the time history of the effort in response to a Heaviside kinematic loading. For a pure extension, $\partial U_{1} / \partial x_{1}(t)=H(t)$, the relaxation of the normal force reads

$$
N_{r}(t)=E|S| H(t)-[(1-2 v) \alpha]^{2} A \int_{-\infty}^{\infty} \exp ^{-i \omega t} \frac{1}{i \omega}\left[\int_{S} \zeta(\omega) d S\right] d \omega
$$

and for pure curvature, $\partial^{2} U_{\alpha} / \partial x_{1}^{2}(t)=H(t)$, the relaxation of the bending reads

$$
M_{\alpha r}(t)=-E I_{\alpha} H(t)+[(1-2 v) \alpha]^{2} A \int_{-\infty}^{\infty} \exp ^{-i \omega t} \frac{1}{i \omega}\left[\int_{S} x_{\alpha} \psi^{\alpha}(\omega) d S\right] d \omega
$$

Conversely, creep functions are the time history of the deformation in response to an Heaviside effort loading. Hence for pure compression, i.e. $N(t)=H(t)$, the creep in extension reads

$$
\frac{\partial U_{1}}{\partial x_{1}}(t)=\int_{-\infty}^{\infty} \exp ^{-i \omega t} \frac{1}{i \omega \widehat{E S}} d \omega
$$

and for pure bending $M_{\alpha}(t)=H(t)$, the creep in curvature reads

$$
\frac{\partial^{2} U_{\alpha}}{\partial x_{1}^{2}}(t)=-\int_{-\infty}^{\infty} \exp ^{-i \omega t} \frac{1}{i \omega \widehat{E I}_{\alpha}} d \omega
$$

\section{Analysis of the poroelastic beam behavior}

The poroelastic effects are hereafter examined according to the mechanical parameters, the boundary conditions, and the frequency.

\subsection{Vanishing poroelastic effect}

The beam behavior laws (38)-(39) show that two situations lead to negligible effects of poroelasticity.

Incompressible porous matrix: As the pressure results from the porous matrix compressibility, when the matrix tends to be incompressible $(1-2 v \rightarrow 0)$, the pressure, then the poroelastic effect tend to vanish in (38)-(39).

Very compressible fluid compared to the porous matrix: If $O\left(E / K_{\mathrm{f}}\right) \gg 1$ then $O(E / A) \gg 1$, since

$$
\frac{1}{A}=\frac{\alpha-\phi}{K_{\mathrm{s}}}+\frac{\phi}{K_{\mathrm{f}}}+\frac{\alpha^{2}}{\lambda+\mu} .
$$

Consequently, the poroelastic effect vanishes in (38)-(39). Moreover, as the pressure gradient becomes negligible in the force balance, $\tilde{u}^{2} \rightarrow 0$, and in turn $\psi^{\alpha}$ and $\zeta$ tend to the solutions of the uncoupled set:

$$
\begin{aligned}
& \frac{\mathcal{K} A}{i \omega \eta} \Delta_{y}(p)-\frac{A}{M} p=V, \text { in } S ; V=1 \text { for } \zeta, \quad V=-y_{\alpha} \text { for } \psi^{\alpha} \\
& \frac{\xi}{h} \cdot p+\underline{\operatorname{grad}}(p) \cdot \underline{n}=0, \quad \text { on } \Gamma
\end{aligned}
$$

\subsection{Fluid effect with impervious boundary condition}

In the no leakage case $(\xi=0)$, the fluid flux, hence the pressure gradient are null on $\Gamma$. Then, integrating over $S(66)$, and (66) multiplied by $y_{\alpha}$, and making the usual integral transformations, the particular pressure fields (denoted here by the index 0 ) satisfy

$$
\frac{A \alpha}{\lambda+\mu} \int_{S} \operatorname{div}_{y}\left(\underline{c}_{0}\right) d s+\frac{A}{M} \int_{S} \zeta_{0} d s=-|S|^{\prime} \quad ; \quad \frac{A \alpha}{\lambda+\mu} \int_{S} y_{\alpha} \operatorname{div}_{y}\left(\underline{b}_{0}^{\alpha}\right) d s+\frac{A}{M} \int_{S} y_{\alpha} \psi_{0}^{\alpha} d s=I_{\alpha}^{\prime}
$$


and, from the identities (74), (75), one obtains

$$
\int_{S} \zeta_{0} d s=-|S|^{\prime}, \quad \int_{S} y_{\alpha} \psi_{0}^{\alpha} d s=I_{\alpha}^{\prime}
$$

consequently, with the usual variables, the beam constitutive laws become

$$
\begin{aligned}
& N=E|S|\left[1+[(1-2 v) \alpha]^{2} \frac{A}{E}\right] \frac{\partial U_{1}}{\partial x_{1}} \\
& M_{\alpha}=-E I_{\alpha}\left[1+[(1-2 v) \alpha]^{2} \frac{A}{E}\right] \frac{\partial^{2} U_{\alpha}}{\partial x_{1}^{2}}
\end{aligned}
$$

Hence, impervious boundary conditions cancel the effect of fluid transfer. The beam behavior remains purely elastic, even if the saturating fluid stiffen the apparent Young modulus trough the effective bulk effect characterized by $[(1-2 v) \alpha]^{2} A$ (which corresponds to the elastic coefficients of the undrained porous medium $\lambda+\alpha^{2} M, \mu$ ).

\subsection{The different poroelastic regimes according to frequency}

From the situation described by the set (64)-(67), different poroelastic regimes can be identified. Recall that at pores scale the flow is dominated by viscous effect as $\omega / \omega_{c} \leq O(\varepsilon)$. As mentioned in Section 4.1.3, the nature of fluid transfer at section scale is related to the dimensionless frequency $\omega\left(\eta h^{2}\right) /(\mathcal{K} A)$. As $A=O\left(A_{c}\right)$, more physical insight is provided by the usual consolidation length $\delta$ associated with elasto-diffusion phenomena:

$$
\delta^{2}=\frac{\mathcal{K} A_{c}}{i \omega \eta}
$$

since the relative order of the transfer and compression terms in (66) depends on the dimensionless ratio $h / \delta$. Moreover, as $\delta$ varies with the frequency, it is convenient to introduce the intrinsic length $\delta_{c}$, which is of the order of $\delta\left(\omega_{c}\right)$, by

$$
\delta_{c}=\frac{\mathcal{K}}{\eta} \sqrt{\frac{A_{c} \rho_{\mathrm{f}} \tau_{\infty}}{\phi}} \text { thus } O\left(\frac{h}{|\delta|}\right)=\frac{h}{\delta_{c}} \frac{\omega}{\omega_{c}}
$$

\subsubsection{Transfer and compression of the same magnitude}

When $h /|\delta|=\left(h / \delta_{c}\right)\left(\omega / \omega_{c}\right)=O(1)$ both transfer and compression terms in (66) are of the same order at the scale of the beam section. As $\omega / \omega_{c} \ll 1$, this situation occurs for beam section such that $h / \delta_{c} \gg 1$. As for the magnitude of the Darcy motion, in this regime $O(\zeta)=O(1)$ and $O\left(\psi^{\alpha}\right)=O(h)$, and expressions (32)-(33) lead to the following estimates:

$$
O\left[\phi\left(\underline{u}_{\mathrm{f}}-\underline{u}\right)_{\alpha}\right]=(1-2 v) \alpha \frac{\delta^{2}}{h^{2}}\left[O\left(\varepsilon^{2} U_{\alpha}^{0}\right)+O\left(\varepsilon U_{1}^{1}\right)\right], \quad O\left[\phi\left(\underline{u}_{\mathrm{f}}-\underline{u}\right)_{1}\right]=\varepsilon O\left[\phi\left(\underline{u}_{\mathrm{f}}-\underline{u}\right)_{\alpha}\right]
$$

Now, if $h /|\delta| \gg 1$ or $\ll 1$ the phenomena at the scale of the beam section are respectively dominated either by the transfer or by the compression mechanism. These situations enable simple assessment of the poroelastic effect.

\subsubsection{Negligible transfer}

When $h /|\delta|=\left(h / \delta_{c}\right)\left(\omega / \omega_{c}\right) \geq O\left(\varepsilon^{-1}\right)$ the transfer becomes negligible and (66) tends to

$$
-\frac{A}{M} p-\alpha \frac{A}{\lambda+\mu} \operatorname{div}_{\mathrm{y}}(\underline{u})=V
$$

Consequently, the relations (63) established for impervious conditions also apply and the beam laws tend to those obtained in the impervious case (45)-(46). Notice however that the pressure distributions for $h / \delta \gg 1$ and for $\xi=0$ are not identical. The pressure boundary conditions when $h / \delta \gg 1$ are matched by boundary layers (depending on $\xi$ ) of thickness $|\delta|$. Outside of these layers, the flow vanishes and the fluid acts as a purely compressible elastic constituent through its bulk modulus, as when $\xi=0$.

As for the magnitude of the Darcy motion, we still have in this regime $O(\zeta)=O(1)$ and $O\left(\psi^{\alpha}\right)=O(h)$, however the gradient of $\zeta$ is limited to the boundary layers. Consequently, from expressions (32)-(33) we deduce:

$$
\begin{aligned}
& O\left[\phi\left(\underline{u}_{\mathrm{f}}-\underline{u}\right)_{\alpha}\right]=(1-2 v) \alpha \frac{\delta}{h}\left[\frac{\delta}{h} O\left(\varepsilon^{2} U_{\alpha}^{0}\right)+O\left(\varepsilon U_{1}^{1}\right)\right] \\
& O\left[\phi\left(\underline{u}_{\mathrm{f}}-\underline{u}\right)_{1}\right]=(1-2 v) \alpha \frac{\delta^{2}}{h^{2}}\left[O\left(\varepsilon^{3} U_{\alpha}^{0}\right)+O\left(\varepsilon^{2} U_{1}^{1}\right)\right]
\end{aligned}
$$




\subsubsection{Negligible compression effect}

This situation occurs when $h /|\delta|=\left(h / \delta_{c}\right)\left(\omega / \omega_{c}\right) \leq O(\varepsilon)$, hence for beam section such that $h / \delta_{c} \geq O(1)$. In that case (66) reduces to

$$
\frac{\mathcal{K} A}{i \omega \eta} \Delta_{y}(p)=V
$$

therefore, the particular fields $\zeta$ and $\psi^{\alpha}$ are respectively $O\left(h^{2} /|\delta|^{2}\right)$ and $O\left(h \cdot h^{2} /|\delta|^{2}\right)$. Consequently:

$$
\int_{S} \zeta d s=-\left|S^{\prime}\right| \frac{i \omega \eta}{\mathcal{K} A}\left|S^{\prime}\right| c_{N}, \quad \int_{S} y_{\alpha} \psi^{\alpha} d s=\left|S^{\prime}\right| \frac{i \omega \eta}{\mathcal{K} A} I_{\alpha}^{\prime} c_{M_{\alpha}}
$$

where $c_{N}=O(1)$ and $c_{M_{\alpha}}=O(1)$ are real positive form coefficients depending on the geometry of the section and on the leakage coefficient $\xi$. Using the usual variables, the poroelastic beam parameters take the form of elastic beam parameters with small poroelastic corrector (the pressure is in phase quadrature with the solid motion and the flow acts as viscous dampers, different under compression and under bending):

$$
\begin{aligned}
& \widehat{E S}=E|S|\left[1+[(1-2 v) \alpha]^{2} \frac{i \omega \eta|S|}{\mathcal{K} E} c_{N}\right] \\
& \widehat{E I_{\alpha}}=E I_{\alpha}\left[1+[(1-2 v) \alpha]^{2} \frac{i \omega \eta|S|}{\mathcal{K} E} c_{M_{\alpha}}\right]
\end{aligned}
$$

In this regime, since $O(\zeta)=O\left(h^{2} /|\delta|^{2}\right)$ and $O\left(\psi^{\alpha}\right)=O\left(h \cdot h^{2} /|\delta|^{2}\right)$, the magnitude of the Darcy motion deduced from (32)-(33) becomes

$$
O\left[\phi\left(\underline{u}_{\mathrm{f}}-\underline{u}\right)_{\alpha}\right]=(1-2 v) \alpha\left[O\left(\varepsilon^{2} U_{\alpha}^{0}\right)+O\left(\varepsilon U_{1}^{1}\right)\right], \quad O\left[\phi\left(\underline{u}_{\mathrm{f}}-\underline{u}\right)_{1}\right]=\varepsilon O\left[\phi\left(\underline{u}_{\mathrm{f}}-\underline{u}\right)_{\alpha}\right]
$$

Remark. The analysis on frequency has to be complemented by the restriction imposed by the axial versus lateral scale separation requirement, as specified in Section 4.2.

\subsection{Approximated pressure distribution}

When $h /|\delta|=O(1)$ no simple expression of the pressure is available. Nevertheless an approximated solution (denoted by ante exponent ${ }^{a}$ ) can be derived as proposed by Scherer et al. (2009). The idea is to consider that $\Gamma \mathrm{r}\left(\widehat{\underline{\Sigma}}_{s}^{1}\right)=0$ instead of $\int_{S} \operatorname{Tr}\left(\widehat{\underline{\Sigma}}_{s}^{1}\right) d s=0$ as established in (62). Thus, for the approximate solution, one has

$$
(\lambda+\mu) \operatorname{div}_{y}\left({ }^{\mathrm{a}} \underline{u}^{2}\right)=\alpha \cdot{ }^{\mathrm{a}} p
$$

This assumption leads to disregard the rotational part of the solid displacement in the equilibrium of the skeleton. Consequently, the initial set becomes uncoupled, and the approximated problem:

$$
\begin{aligned}
& \frac{\mathcal{K} A}{i \omega \eta} \Delta_{y}\left({ }^{\mathrm{a}} p\right)-{ }^{\mathrm{a}} p=V \text { in } S, \quad V=1 \text { for }{ }^{\mathrm{a}} \zeta, \quad V=-y_{\alpha} \text { for }{ }^{\mathrm{a}} \psi_{\alpha} \\
& \frac{\xi}{h} \cdot{ }^{\mathrm{a}} p+\underline{\operatorname{grad}}\left({ }^{\mathrm{a}} p\right) \cdot \underline{n}=0 \quad \text { on } \Gamma
\end{aligned}
$$

enables easier determination of ${ }^{\mathrm{a}} \psi^{\alpha}$ and $\zeta$ through standard solving methods. Note that this problem introduces an approximated length of the elasto-diffusion phenomena, $\delta_{a}$ defined by

$$
\left(\delta_{a}\right)^{2}=\frac{\mathcal{K} A}{i \omega \eta}=\frac{A}{A_{c}} \delta^{2} \quad \text { with } \frac{1}{2} \leq \frac{A}{A_{c}}=\left(1+\frac{1-2 v}{1+\frac{\lambda+2 \mu}{\alpha^{2} M}}\right)^{-1} \leq 1
$$

Hence, the maximum discrepancy between $\delta_{a}$ and $\delta$ corresponds to a ratio of $\sqrt{2}$, reached for $v \rightarrow 0$ and $\lambda+2 \mu \ll \alpha^{2} M$. Note also the similarity of the problem governing the approximate solutions of ${ }^{\mathrm{a}} \zeta$ and ${ }^{\mathrm{a}} \psi^{\alpha}$ with the problem (47)-(48) obtained for very compressible fluid.

\section{Exact analytical solutions: flat and circular sections}

The beam parameters depend on the local fields solution of the in-plane poroelastic problems (64)-(67). In general, this latter problems have to be solved numerically. However, for flat and circular sections exact solutions can be established. As they correspond to two extreme geometry, they are useful as reference solutions and to assess the validity of approximated solutions. 


\subsection{Flat sections}

When the field in the section tends to depend on a single variable, e.g. $y_{3}$, the problem tends to be 1D (disregarding the $y_{2}$-dependency). This situation is reached for sections more elongated in the direction $\underline{a}_{2}$ than along $\underline{a}_{3}\left(-h / 2 \leq y_{3} \leq h / 2\right)$ and corresponds to poroelastic plates as studied by Taber (1992). Zhang and Cowin (1994) also suggest that 1D solution could approach the situation of rectangular section having free flow boundary condition on $y_{3}= \pm h$ and impervious conditions in the other faces. In 1D case, (dropping the indice 3) the dimensionless problem (64)-(67) defining $\zeta$ and $\psi$ reduces to (' and " stand for the simple and second derivative according $y$ ):

$$
\begin{aligned}
& 2(1-v) u^{\prime \prime}-\alpha p^{\prime}=0 \text { for }|y| \leq h / 2, \quad 2(1-v) u^{\prime}=\alpha p \text { on } y= \pm h / 2 \\
& \frac{\mathcal{K} A}{i \omega \eta} p^{\prime \prime}-\frac{A}{M} p-\alpha \frac{A}{\lambda+\mu} u^{\prime}=V \text { for }|y| \leq h / 2, \quad \frac{\xi}{h} p=\mp p^{\prime} \text { on } y= \pm h / 2
\end{aligned}
$$

From (51) one deduces that $2(1-v) u^{\prime}=\alpha p$. This expression reported in $(52-a)$ provides the pressure equation:

$$
\frac{\mathcal{K} A}{i \omega \eta} p^{\prime \prime}-\frac{A}{A_{c}} p=V, \quad V=1 \text { for } \zeta, \quad V=-y \text { for } \psi
$$

The integration of this latter with the boundary conditions (52-b), leads to $\left(\delta^{2}=\mathcal{K} A_{c} / i \omega \eta\right)$ :

$$
\begin{aligned}
& \zeta=\frac{A_{c}}{A}\left[\frac{\cosh \left(\frac{y}{\delta}\right)}{\cosh \left(\frac{h}{2 \delta}\right)+\sinh \left(\frac{h}{2 \delta}\right) \frac{h}{\xi \delta}}-1\right] \\
& \psi=-\frac{A_{c}}{A}\left[\left(1+\frac{2}{\xi}\right) \frac{\sinh \left(\frac{y}{\delta}\right)}{\sinh \left(\frac{h}{2 \delta}\right)+\cosh \left(\frac{h}{2 \delta}\right) \frac{h}{\xi \delta}}-\frac{2 y}{h}\right] \frac{h}{2}
\end{aligned}
$$

and, by further integration we deduce the following beam parameters (as a consequence of the 1D geometry of the section, $E$ and $[(1-2 v) \alpha]^{2}$ are respectively changed into $E /\left(1-v^{2}\right)$ and $\left.[((1-2 v) \alpha) /(1-v)]^{2}\right)$ :

$$
\begin{aligned}
& \widehat{E S}=\frac{E|S|}{1-v^{2}}\left[1+[(1-2 v) \alpha]^{2} \frac{1+v}{1-v} \frac{A_{c}}{E} \mathcal{F}_{\mathcal{S}}\right], \quad \mathcal{F}_{\mathcal{S}}=1-\frac{2 \delta / h}{\operatorname{coth}\left(\frac{h}{2 \delta}\right)+\frac{h}{\xi \delta}} \\
& \widehat{E I}=\frac{E I}{1-v^{2}}\left[1+[(1-2 v) \alpha]^{2} \frac{1+v}{1-v} \frac{A_{c}}{E} \mathcal{F}_{\mathcal{I}}\right], \quad \mathcal{F}_{\mathcal{I}}=1-6\left(1+\frac{2}{\xi}\right) \frac{\delta}{h} \frac{-\frac{2 \delta}{h}+\operatorname{coth}\left(\frac{h}{2 \delta}\right)}{1+\operatorname{coth}\left(\frac{h}{2 \delta}\right) \frac{h}{\xi \delta}}
\end{aligned}
$$

The parameters simplify for impervious condition into

$$
\widehat{E S} / E|S|=\widehat{E I} / E I=\frac{1}{1-v^{2}}\left[1+[(1-2 v) \alpha]^{2} \frac{1+v}{1-v} \frac{A_{c}}{E}\right]
$$

and for free flow condition into

$$
\begin{aligned}
& \widehat{E S}=\frac{E|S|}{1-v^{2}}\left[1+[(1-2 v) \alpha]^{2} \frac{1+v}{1-v} \frac{A_{c}}{E}\left[1-\frac{2 \delta}{h} \operatorname{th}\left(\frac{h}{2 \delta}\right)\right]\right] \\
& \widehat{E I}=\frac{E I}{1-v^{2}}\left[1+[(1-2 v) \alpha]^{2} \frac{1+v}{1-v} \frac{A_{c}}{E}\left[1-6 \frac{\delta}{h}\left(-\frac{2 \delta}{h}+\operatorname{coth}\left(\frac{h}{2 \delta}\right)\right)\right]\right]
\end{aligned}
$$

In this latter free flow case, using the residue method for inversion (Spiegel, 1965), the relaxation functions in compression and bending read

$$
\begin{aligned}
& N_{r}(t>0)=\frac{E|S|}{1-v^{2}}\left[1+[(1-2 v) \alpha]^{2} \frac{1+v}{1-v} \frac{A_{c}}{E} \sum_{1}^{\infty} 2\left[\frac{2}{(2 n-1) \pi}\right]^{2} \exp \left(-t / \tau_{n}\right)\right], \quad \tau_{n}=\frac{\eta h^{2}}{4 \mathcal{K} A_{c}}\left[\frac{(2 n-1) \pi}{2}\right]^{2} \\
& M_{r}(t>0)=-\frac{E I}{1-v^{2}}\left[1+[(1-2 v) \alpha]^{2} \frac{1+v}{1-v} \frac{A_{c}}{E} \sum_{1}^{\infty} 6\left[\frac{1}{n \pi}\right]^{2} \exp \left(-t / \tau_{n}^{\prime}\right)\right], \quad \tau_{n}^{\prime}=\frac{\eta h^{2}}{4 \mathcal{K} A_{c}}[n \pi]^{2}
\end{aligned}
$$


Recalling that

$$
2 \sum_{1}^{\infty}\left[\frac{2}{(2 n-1) \pi}\right]^{2}=6 \sum_{1}^{\infty}\left[\frac{1}{n \pi}\right]^{2}=1
$$

the instantaneous compression and bending responses are that of a beam with an apparent modulus

$$
E+\frac{[(1-2 v) \alpha]^{2}}{1-v} A
$$

The long term responses are that of the elastic beam without pressure effect (modulus $E$ ). Despite these similarities, the time response of both mechanisms differs: the characteristic decay time in bending is longer $\left(\tau_{1}^{\prime}=4 \tau_{1}\right)$ than in compression. Whatever the leakage condition, the same method would enable to obtain analytical expressions for relaxation (and creep). The general form is a series of decaying exponential, the discrete characteristic times of which are determined by the poles of the considered functions.

These results are illustrated in Fig. 2, where the dimensionless complex parts, $\mathcal{F}_{\mathcal{S}}, \mathcal{F}_{\mathcal{I}}$ of the compression and bending modulus (58)-(59), are drawn versus the dimensionless frequency $w=\omega /\left[4 \mathcal{K} A_{c} / \eta h^{2}\right]$ in the free flow case $(\xi=\infty)$, and with leakage boundary condition (taking $\xi=10$ ). The difference of frequency responses between compression and bending is clearly evidenced, as well as the influence of the flux condition. Fig. 3 depicts free flow dimensionless time responses derived from the Fourier transforms of $\mathcal{F}_{\mathcal{S}}, \mathcal{F}_{\mathcal{I}}$ (with $\xi=\infty$ ), as function of the dimensionless time $t^{\prime}=t /\left[\eta h^{2} / 4 \mathcal{K} A_{c}\right]$. It confirms the much longer time decay in bending than in compression.

\subsection{Circular sections}

The principle of the resolution presented hereafter applies for any uniform leakage condition. For simplicity, we only focus on free flow condition. Because of the circular geometry of the section of radius $R$, the problems are investigated using the polar variables $(r, \theta)$.
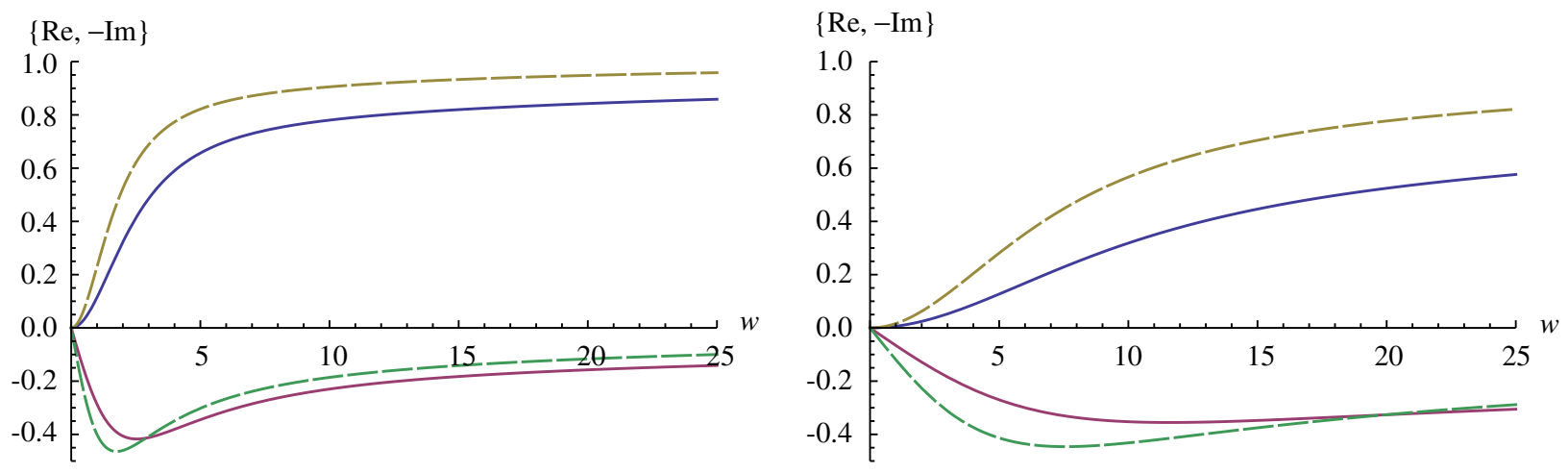

Fig. 2. Flat sections. Dimensionless complex parts, $\mathcal{F}_{\mathcal{S}}, \mathcal{F}_{\mathcal{I}}$, (see (58)-(59) of the modulus of compression $\widehat{E S}$ (left) and bending $\widehat{E I}$ (right) versus the dimensionless frequency $w=\omega /\left(4 \mathcal{K} A_{c} / \eta h^{2}\right)$. Both real and imaginary parts are positive, but the opposite of the imaginary part is plotted to facilitate the lecture. Solid lines correspond to the free flow case $(\xi=\infty)$, dashed lines to leakage boundary condition $(\xi=10)$.

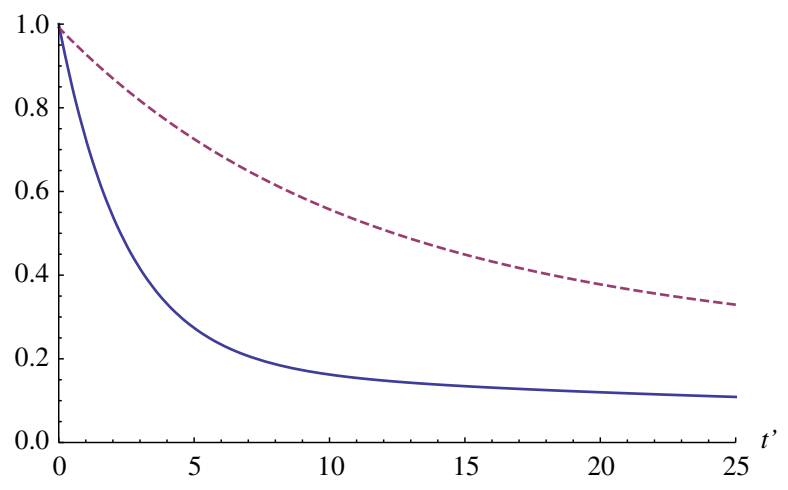

Fig. 3. Flat sections. Dimensionless time response deduced from the Fourier transforms of $\mathcal{F}_{\mathcal{S}}, \mathcal{F}_{\mathcal{I}}$. Solid line: compression. Dashed line: bending. Dimensionless time $t^{\prime}=t /\left[\eta h^{2} / 4 \mathcal{K} A_{c}\right]$. 
6.2.1. Compression

As the section is circular and the loading uniform in compression, the solution of the in-plane poroelastic problem (64)-(67) is necessarily on the radial form:

$$
p=p(r), \quad \underline{u}=u(r) \underline{e}_{r}
$$

Hence, denoting by ' the $r$-derivative, and dropping the $y$-indice:

$$
\begin{aligned}
& \underline{\operatorname{grad}}(p)=p^{\prime} \underline{e}_{r}, \quad \Delta(p)=\frac{1}{r}\left(r p^{\prime}\right)^{\prime} \\
& \underline{\underline{\mathrm{e}}}_{s}(\underline{u})=u^{\prime} \underline{e}_{r} \otimes \underline{e}_{r}+\frac{u}{r} \underline{e}_{\theta} \otimes \underline{e}_{\theta}, \quad \underline{\operatorname{grad}}(\operatorname{div}(\underline{u}))=\Delta(\underline{u})=\left[\frac{(r u)^{\prime}}{r}\right]^{\prime} \underline{e}_{r}=\left[\Delta(u)-\frac{u}{r^{2}}\right]^{\prime} \underline{e}_{r}
\end{aligned}
$$

Introducing these expressions in the set (70)-(72) with $V=1$ and $\xi=\infty$ leads to

$$
\begin{aligned}
& \underline{\operatorname{grad}}[2(1-v) \operatorname{div}(\underline{u})-\alpha p]=\underline{0} \text { for } 0 \leq r \leq R \\
& \frac{\mathcal{K} A}{i \omega \eta} \Delta(p)-\frac{A}{M} p-\alpha \frac{A}{\lambda+\mu} \operatorname{div}(\underline{u})=1 \text { for } 0 \leq r \leq R
\end{aligned}
$$

The first equation gives $\operatorname{div}(\underline{u})=\left(\alpha p+c_{0}\right) /[2(1-v)]$, where $c_{0}$ is a constant, and consequently the pressure is governed by

$$
\frac{\mathcal{K} A}{i \omega \eta} \Delta_{y}(p)-\frac{A}{A_{c}} p=1+\alpha \frac{A}{\lambda+2 \mu} c_{0}
$$

Recalling that $\delta^{2}=\left(\mathcal{K} A_{c}\right) /(i \omega \eta)$, the solution finite in $r=0$ and null in $r=R$ reads

$$
p(r)=\frac{A_{c}}{A}\left[1+\alpha \frac{A}{\lambda+2 \mu} c_{0}\right]\left[-1+\frac{J_{0}(i r / \delta)}{J_{0}(i R / \delta)}\right]
$$

where $J_{0}$ is the Bessel function of zero order. To identify the constant $c_{0}$ we use the first equality of (69) which reduces to

$$
\int_{0}^{R}\left(\alpha p+c_{0}\right) r d r=2(1-v) \alpha \int_{0}^{R} p r d r
$$

and gives, using the relation $\int_{0}^{z} z J_{0}(z) d z=J_{1}(z)$ :

$$
c_{0}=\alpha(1-2 v) \frac{\int_{0}^{R} r p d r}{\int_{0}^{R} r d r}=\frac{A_{c}}{A}\left[1+\alpha \frac{A}{\lambda+2 \mu} c_{0}\right]\left[-1+\frac{2 \delta J_{1}(i R / \delta)}{i R} J_{0}(i R / \delta)\right]
$$

The resolution of this linear equation provides the pressure function (and incidentally the solid displacement). Finally, after integration and some algebra, the exact poroelastic compression modulus for circular section is

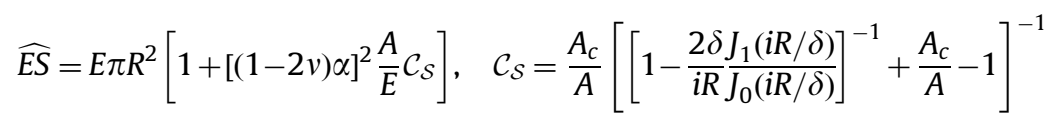

Noticing that the limit values at high and low frequency are respectively given by Abramowitz and Stegun (1964):

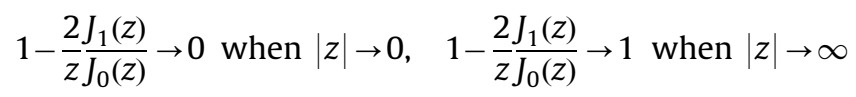

we retrieve that the instantaneous (undrained) and long term (drained) moduli are respectively $E+[(1-2 v) \alpha]^{2} A$ and $E$.

For comparison, the approximate solution obtained by integrating (48) for circular section gives

$$
\widehat{E S^{a}}=E \pi R^{2}\left[1+[(1-2 v) \alpha]^{2} \frac{A}{E} \mathcal{C}_{\mathcal{S}}^{a}\right], \quad \mathcal{C}_{\mathcal{S}}^{a}=1-\frac{2 \delta_{a} J_{1}\left(i R / \delta_{a}\right)}{i R} \frac{J_{0}\left(i R / \delta_{a}\right)}{2}
$$

This approximation respects the same instantaneous and long term moduli values than the exact solution. The approximation is closer to the exact value as $v \rightarrow 1 / 2$ (hence $A_{c} \rightarrow A$ ) while the maximum discrepancy is obtained when $v \rightarrow 0$ and $A_{c} \rightarrow 2 A$. The corresponding approximated relaxation function reads

$$
N_{r}^{a}(t>0)=E|S|\left[1+[(1-2 v) \alpha]^{2} \frac{A}{E} \sum_{1}^{\infty} 4 \frac{\exp \left(-t / \tau_{a n}\right)}{\lambda_{0, n}^{2}}\right], \quad \tau_{a n}=\frac{\eta R^{2}}{\mathcal{K} A} \lambda_{0, n}^{2}
$$

where the $\lambda_{0, n}$ are the (simple and real) zero of $J_{0}\left(\lambda_{0,1} \approx 2.405, \lambda_{0,2} \approx 5.520, \lambda_{0,3} \approx 8.654 \ldots\right)$. Note that, for the exact solution, the discrete time series $\tau_{n}$ is related to the poles of $\widehat{E S}$, i.e.

$$
\tau_{n}=\frac{\mathcal{K} A_{c}}{\eta}\left[\frac{\lambda_{n}}{R}\right]^{2}
$$


where $\lambda_{n}$ are the roots of the equation

$$
\frac{z J_{0}(z)}{2 J_{1}(z)}=1-\frac{A}{A_{c}}
$$

\subsubsection{Bending}

The solution of the in-plane poroelastic problem (64)-(67) necessarily involves the bending direction e. However, for circular section, any rotation of $\underline{\mathbf{e}}$, imposes the same rotation of the solution. Consequently according to the tensor functions representation theory (Boehler, 1987) the local fields are of the following form:

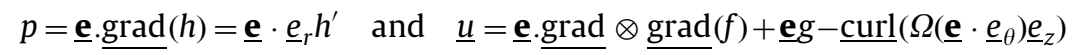

where the potential functions $f, g, h, \Omega$ depends on $r$ only. These expressions introduced in the momentum balance (64) lead to the following vectorial differential equation:

$$
\underline{\mathbf{e}}\left[\frac{\mathcal{F}^{\prime}}{r}+(1-2 v)\left[\Delta(g)-F^{\prime}\right]\right]+\underline{r}\left(\underline{\mathbf{e}} \cdot \underline{e}_{r}\right)\left[\frac{\mathcal{F}^{\prime}}{r}+(1-2 v) \frac{F}{r}\right]^{\prime}=\underline{0}
$$

with

$$
\mathcal{F}=2(1-v) \Delta(f)+g-\alpha h \text { and } F=-\left[\frac{(\Omega r)^{\prime}}{r}\right]^{\prime}
$$

Since $\underline{\mathbf{e}}$ and $\underline{r}$ are independent vectors, both components of (57) vanishes. Form the $\underline{r}$-component we deduce by integration ( $c_{1}$ is a constant):

$$
\mathcal{F}^{\prime}+(1-2 v) F=2 c_{1} r
$$

This result, introduced in the e-component yields after integration (accounting for finite value on $r=0$ of the pressure $h^{\prime} \cos (\theta)$ and the volume variation $\left.\operatorname{div}(\underline{u})=(\Delta(f)+g)^{\prime} \cos (\theta)\right)$ :

$$
(1-2 v)\left(g^{\prime}-F\right)=-c_{1} r
$$

Combining these two results with the expression of $\mathcal{F}$, one obtains

$$
[\Delta(f)+g]^{\prime}=\frac{1}{2(1-v)}\left(\alpha h^{\prime}+c_{1} r\right)
$$

This relation enables to identify the pressure function $h^{\prime}$ : considering the mass balance equation (65) with the source associated to bending $V=-y=-\underline{\mathbf{e}} \cdot \underline{r}$, we have

$$
\underline{\mathbf{e}} \cdot \underline{e}_{r}\left[\frac{\mathcal{K} A}{i \omega \eta} \Delta(h)-\frac{A}{M} h-\alpha \frac{A}{\lambda+\mu}(\Delta(f)+g)\right]^{\prime}=-\underline{\mathbf{e}} \cdot \underline{r}
$$

With the previous results, this equation simplifies into

$$
\left[\frac{\mathcal{K} A}{i \omega \eta} \Delta(h)-\frac{A}{A_{c}} h\right]^{\prime}=r\left(-1+c_{1} \frac{\alpha A}{\lambda+2 \mu}\right)
$$

The solution $h^{\prime}$ having a finite value on $r=0$ and respecting the zero pressure condition on $r=R\left(\right.$ i.e. $\left.h^{\prime}(R)=0\right)$ is, where $J_{1}$ is the Bessel function of first order:

$$
h^{\prime}=\frac{A_{c}}{A}\left[-1+c_{1} \frac{\alpha A}{\lambda+2 \mu}\right]\left[r-R \frac{J_{1}(i r / \delta)}{J_{1}(i R / \delta)}\right]
$$

Now, $c_{1}$ is deduced from the second equality of (68) which reads in the present case:

$$
\int_{S} r \cos ^{2}(\theta)(\Delta(f)+g)^{\prime} r d r d \theta=\alpha \int_{S} r \cos ^{2}(\theta) h^{\prime} r d r d \theta
$$

and gives, using the relation $\int_{0}^{z} z^{2} J_{1}(z) d z=z^{2} J_{2}(z)$ :

$$
c_{1}=\alpha(1-2 v) \frac{\int_{0}^{R} r^{2} h^{\prime} d r}{\int_{0}^{R} r^{3} d r}=\frac{A_{c}}{A}\left[-1+c_{1} \frac{\alpha A}{\lambda+2 \mu}\right]\left[1-\frac{4 \delta}{i R} \frac{J_{2}(i R / \delta)}{J_{1}(i R / \delta)}\right]
$$

The resolution of this linear equation closes the determination of the pressure function. The determination of the displacement field, not necessary for the sequel, is not reported.

Finally, the exact poroelastic bending modulus of circular sections, is given by

$$
\widehat{E I}=E \frac{\pi}{4} R^{4}\left[1+[(1-2 v) \alpha]^{2} \frac{A}{E} \mathcal{C}_{\mathcal{I}}\right], \quad \mathcal{C}_{\mathcal{I}}=\frac{A_{c}}{A}\left[\left[1-\frac{4 \delta}{i R} \frac{J_{2}(i R / \delta)}{J_{1}(i R / \delta)}\right]^{-1}+\frac{A_{c}}{A}-1\right]^{-1}
$$


Noticing that

$1-\frac{4}{z} \frac{J_{2}(z)}{J_{1}(z)} \rightarrow 0$ when $|z| \rightarrow 0, \quad 1-\frac{4 J_{2}(z)}{z} \frac{J_{1}(z)}{L_{1}} \rightarrow 1$ when $|z| \rightarrow \infty$

the instantaneous $(\delta / R \rightarrow 0)$ and long term $(\delta / R \rightarrow \infty)$ behavior are respectively governed by the undrained modulus $E+[(1-2 v) \alpha]^{2} A$ and the drained modulus $E$.

For comparison, the approximated solution is derived from the approximated mass balance equation (48) with the source associated to bending $V=-y=-\underline{E} \cdot \underline{r}$. It reads, with similar notations:

$$
\left[\frac{\mathcal{K} A}{i \omega \eta} \Delta\left({ }^{a} h\right)-{ }^{a} h\right]^{\prime}=-r
$$

Then, after calculations, the approximated poroelastic bending modulus is given by

$$
\widehat{E I^{a}}=E \frac{\pi}{4} R^{4}\left[1+[(1-2 v) \alpha]^{2} \frac{A}{E} \mathcal{C}_{\mathcal{I}}^{a}\right], \quad \mathcal{C}_{\mathcal{I}}^{a}=1-\frac{4 \delta_{a}}{i R} \frac{J_{2}\left(i R / \delta_{a}\right)}{J_{1}\left(i R / \delta_{a}\right)}
$$

Note again that the approximation respects the instantaneous and long term moduli and is closer to the exact value as $v$ tends to $1 / 2$ (as under compression). The corresponding approximated bending relaxation function derived by inversion reads

$$
M_{r}^{a}(t>0)=-E \frac{\pi R^{4}}{4}\left[1+[(1-2 v) \alpha]^{2} \frac{A}{E} \sum_{1}^{\infty} 8 \frac{\exp \left(-t / \tau_{a n}^{\prime}\right)}{\lambda_{1, n}^{2}}\right], \quad \tau_{a n}^{\prime}=\frac{\eta R^{2}}{\mathcal{K} A} \lambda_{1, n}^{2}
$$

where the $\lambda_{1, n}$ are the (simple and real) zero of $J_{1}\left(\lambda_{1,0} \approx 3.832, \lambda_{1,1} \approx 7.016, \lambda_{1,2} \approx 10.173, \ldots\right)$. These values differ from that given by the exact solution: the time series $\tau_{n}^{\prime}$ related to the poles of $\widehat{E I}$ is

$$
\tau_{n}^{\prime}=\frac{\eta R^{2}}{\mathcal{K} A_{c}} \lambda_{n}^{2}
$$

$\lambda_{n}$ being the roots of the equation

$$
\frac{z J_{1}(z)}{4 J_{2}(z)}=1-\frac{A}{A_{c}}
$$

\subsubsection{Numerical comparisons}

The numerical comparison of the different analytical results enables to estimate the validity of the approximation of Section 5.4. Calculations are performed considering the maximum possible discrepancy reached when $A_{c}=2 A$. Fig. 4 , presents the exact $\left(\mathcal{C}_{\mathcal{S}}, \mathcal{C}_{\mathcal{I}}\right)$ and approximated $\left(\mathcal{C}_{\mathcal{S}}^{a}, \mathcal{C}_{\mathcal{I}}^{a}\right)$ dimensionless complex parts of the compression and bending modulus of circular section (53)-(54), versus the dimensionless frequency $w=\omega /\left[\mathcal{K} A_{c} / \eta R^{2}\right]$. It appears that the approximation $\operatorname{Tr}\left({ }^{a} \underline{\underline{S}}_{s}^{1}\right)=0$ is rather good, at least for circular sections. For comparison the results for the flat section are also given showing that the $2 \mathrm{D}$ (circular) or $1 \mathrm{D}$ (flat) are quite similar provided that, the thickness is the double of the radius $h=2 R$. This illustrates the sensitivity to the geometrical aspect of the section.

\section{Poroelastic beam with visco-inertial inner flow}

We finally investigate the particular situations where the pores scale flow is in visco-inertial regime, i.e. $\omega / \omega_{c}=O(1)$ thus $i \omega \rho_{\mathrm{f}} \mathrm{K} / \eta=O(1)$, while the whole motion inertia remains negligible at the section scale, i.e. $\omega<O\left(h^{-1} \sqrt{E / \rho_{m}}\right)$. This occurs for beam section such that $h<\left(\sqrt{E / \rho_{m}}\right) / \omega_{c}$. With inner inertia of the flow and quasi-statism within the section, the set (9)-(10) leads, after scaling and following the asymptotic process, to identical solutions for the first and second problems, as for pure viscous flows. However, the third problem is modified into

$$
\begin{aligned}
& \underline{\operatorname{div}}_{y}\left(\underline{\underline{\Sigma}}_{\mathrm{s}}^{1}\right)+\frac{i \omega \rho_{\mathrm{f}} \mathrm{K}}{\eta} \underline{\operatorname{grad}}_{y}\left(p^{1}\right)=0 \text { in } S \\
& \underline{\underline{\Sigma}}_{\mathrm{s}}^{1} \cdot \underline{\underline{n}}=0 \text { on } \Gamma \\
& \operatorname{div}_{y}\left(\frac{\mathrm{K}}{i \omega \eta} \underline{\operatorname{grad}}_{y}\left(p^{1}\right)\right)-\frac{P^{1}}{M}-\left(\alpha-\frac{i \omega \rho_{\mathrm{f}} \mathrm{K}}{\eta}\right)\left[\operatorname{div}_{y}\left(\underline{u}^{2}\right)+u_{1, x_{1}}^{1}\right]=0 \text { in } S \\
& \underline{\xi} \cdot p^{1}+\underline{\operatorname{grad}}\left(p^{1}\right) \cdot \underline{n}=0 \text { on } \Gamma
\end{aligned}
$$

Compared with Section 4.1.3, the change due to the visco-inertial flow consists in replacing $\mathcal{K}$ by $\mathrm{K}(\omega)$ and $\alpha$ by the complex parameter $\tilde{\alpha}=\alpha-i \omega \rho_{\mathrm{f}} \mathrm{K} / \eta$ in the mass balance equation. The solution is again decomposed into elastic and poroelastic contributions denoted here by $\tilde{\sim}$, i.e. $\left(\underline{u}^{2}, p^{1}\right)=\left(\underline{u}^{e}, 0\right)+\left(\underline{\tilde{u}}^{2}, \tilde{p}^{1}\right)$. The resolution is reported in Appendix. Finally, 

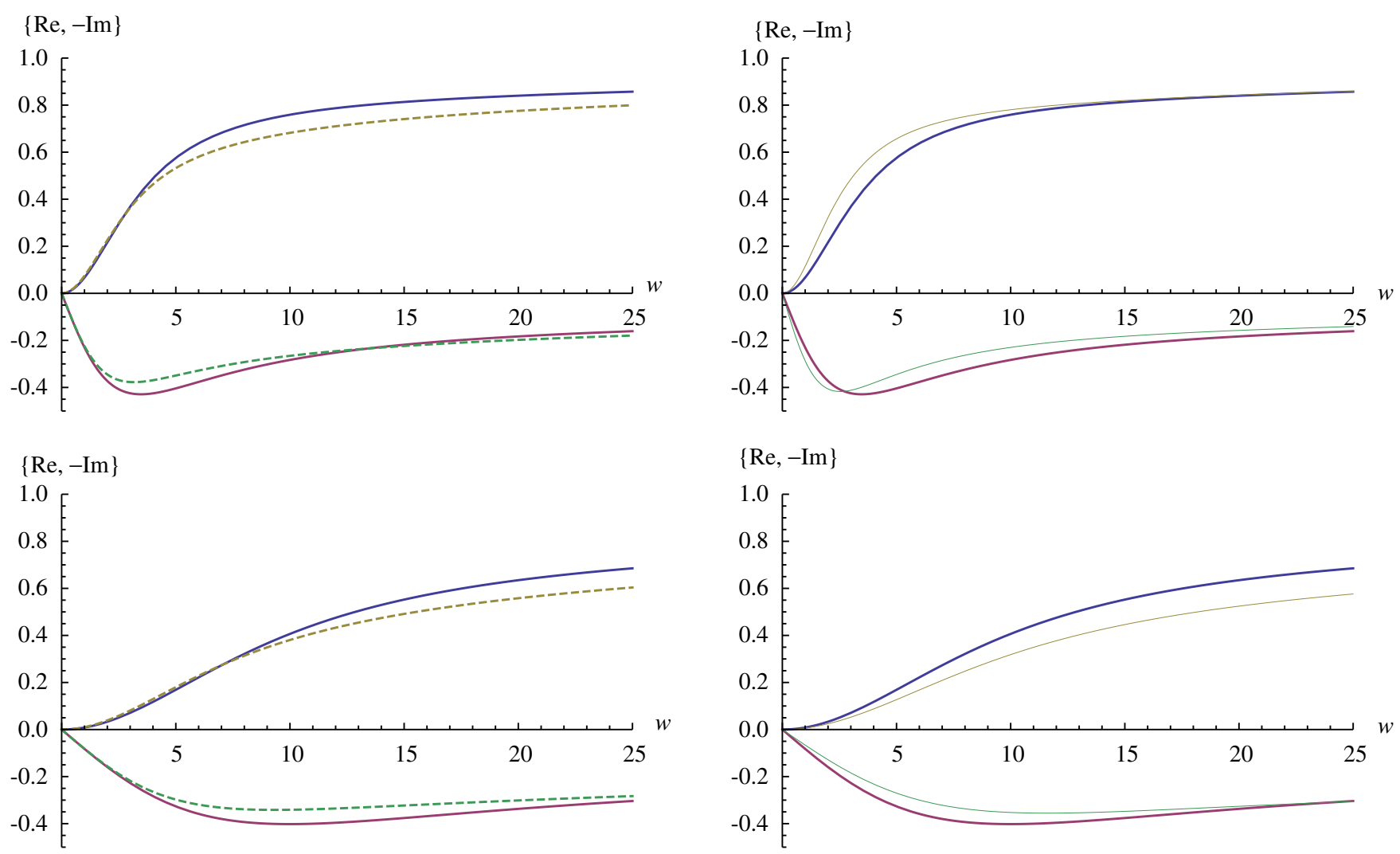

Fig. 4. Circular section with free flow conditions. Dimensionless complex parts of the modulus of compression $\widehat{E S}$ (top) and bending $\widehat{E I}$ (bottom) versus the dimensionless frequency $w=\omega /\left(\mathcal{K} A_{c} / \eta R\right.$ ). Bold solid lines correspond to solutions $\mathcal{C}_{\mathcal{S}}, \mathcal{C}_{\mathcal{I}}(60)$, (65) (real and imaginary parts are positive, the opposite imaginary parts are plotted to facilitate the lecture). On the left, these solutions are compared to the approximated solutions in dashed line $\mathcal{C}_{\mathcal{S}}^{a}$, $\mathcal{C}_{\mathcal{I}}^{a}$ (61), (67), with $A_{c}=2 A$. On the right, solutions $\mathcal{C}_{\mathcal{S}}, \mathcal{C}_{\mathcal{I}}$ for circular section of radius $R$ are compared to the exact solutions for flat section of thickness $h=2 R\left(\mathcal{F}_{\mathcal{S}}\right.$, $\mathcal{F}_{\mathcal{I}}$, drawn in normal solid line).

the dynamic balance equations are established in the same way as previously, with the remark that the inertia term now reads

$$
-\omega^{2}\left[\rho_{m}+\frac{i \omega \rho_{\mathrm{f}} \mathrm{K}}{\eta} \rho_{\mathrm{f}}\right] \underline{u}
$$

cf. (9). Consequently, assuming bi-symmetric sections, the dynamic beam description for visco-inertial regime of inner flow is given by (the expressions of the coefficients are given section)

$$
\begin{aligned}
& \frac{\partial N}{\partial x_{1}}=-\left[\rho_{m}+\frac{i \omega \rho_{\mathrm{f}} \mathrm{K}}{\eta} \rho_{\mathrm{f}}\right] \omega^{2} S U_{1}, \quad N=\tilde{E S} \frac{\partial U_{1}}{\partial x_{1}} \\
& \frac{\partial M_{\alpha}}{\partial x_{1}}-T_{\alpha}=0, \quad M_{\alpha}=-E \tilde{I}_{\alpha} \frac{\partial^{2} U_{\alpha}}{\partial x_{1}^{2}} \\
& \frac{\partial T_{\alpha}}{\partial x_{1}}=-\left[\rho_{m}+\frac{i \omega \rho_{\mathrm{f}} \mathrm{K}}{\eta} \rho_{\mathrm{f}}\right] \omega^{2} S U_{\alpha} \\
& \frac{\partial M_{1}}{\partial x_{1}}=-\rho_{m} \omega^{2} I_{t} \Omega, \quad M_{1}=\mu I_{t} \frac{\partial \Omega}{\partial x_{1}}
\end{aligned}
$$

\section{Conclusion}

The asymptotic approach provides a theoretical frame to analyze the poroelasticity effect on the beam behavior. The method enables a rigorous formulation that account for the magnitude of permeability, the flow conditions on the section periphery, the gas or liquid nature of the fluid, the frequency range of the oscillations. The study mostly focuses on usual situations with inner flow dominated by viscosity, but also investigates less classical situation involving visco-inertial inner flow. The frequency dependent constitutive beam parameters can be computed numerically by solving an in-plane 
poroelastic problem in the section, or analytically for flat or circular sections. Using complex modulus for the solid and/or complex viscosity for the fluid, the results can easily be extended to beams made of viscoelastic constituents. Note however that the description assumes the validity of the Biot model. This imposes a viscous stress in the fluid much smaller than both fluid pressure and elastic stress in the skeleton (Boutin and Auriault, 1990). This which may not be the case for highly viscous fluids in very soft solid matrix (foam, gel).

Except in the degenerated cases of very compressible fluid or impervious periphery of the section, the poroelasticity induces creep and relaxation phenomena in compression and bending but not in torsion (for bi-symmetric sections). Moreover, the characteristic decay time differ for both mechanisms. These specific features differentiate the poroelastic beams from beams made of viscoelastic material.

The theoretical formulation improves the existing analysis by explicitly defining the poroelastic state within the beam. In particular, it is demonstrated that the mean value on the section of the in-plane total stress and its momentum are null. This clarifies the existing approximations assuming either the in-plane stress to be zero (Scherer et al., 2009), or the field to be uni-dimensional (Zhang and Cowin, 1994), and underlines the similarities with the modeling of poroelastic plates (Taber, 1992). Moreover, the exact analytical solutions obtained for flat and circular sections can be used as reference cases for usual geometry (and the same procedure could be applied to different boundary conditions). The discrepancy induced by the approximations are illustrated through numerical comparisons.

The situation of axial diffusion studied by Li et al. (1995) cannot be obtained with the isotropic assumption of permeability used in this paper. However, it could also be derived by the same method by considering a high permeability in the axial direction only. In that case, the pressure would remain an independent variable, conversely to the present study.

Notice finally that more complicated situations as heterogenous or non-straight (but periodic) poroelastic beams, could also be investigated through the asymptotic method. Furthermore expansions at higher orders can also be contemplated to analyze the shear effect (as in Timoshenko beams) for poroelastic rods of limited slenderness.

\section{Appendix A}

\section{A.1. Poroelastic stress-strain state-third problem}

We determine here successively the elastic contribution (drained state) $\left(\underline{u}^{e}, 0\right)$ and the poroelastic contribution $\left(\widehat{\underline{u}}^{2}, p^{1}\right)$, the complete solution being $\left(\underline{u}^{2}, p^{1}\right)=\left(\underline{u}^{e}, 0\right)+\left(\underline{\widehat{u}}^{2}, p^{1}\right)$.

The beam problem in the elastic drained state (variables denoted by ${ }^{e}$ ) reads

$$
\begin{aligned}
& \underline{\underline{\sigma}}_{s}^{e}=2 \mu \underline{\underline{\mathrm{e}}}_{s y}\left(\underline{u}^{e}\right)+\lambda\left[\operatorname{div}_{y}\left(\underline{u}^{e}\right)+u_{1, x_{1}}^{1} \underline{\underline{I}}_{s}, \quad \underline{\operatorname{div}}_{y}\left(\underline{\underline{\sigma}}_{\mathrm{s}}^{e}\right)=\underline{0} \text { in } S\right. \\
& \underline{\underline{\sigma}}_{s}^{e} \cdot \underline{n}=\underline{0} \text { on } \Gamma
\end{aligned}
$$

The solution $\underline{u}^{e}$ under the forcing $u_{1, x_{1}}^{1}$ is classically derived noticing that the strains of the plane fields $\underline{y}$ and $\underline{\xi}^{\alpha}=y_{\alpha} \underline{y}-\frac{1}{2}\|y\|^{2} \underline{a}_{\alpha}$ are respectively $\underline{\underline{\mathrm{e}}}_{s y}(\underline{y})=\underline{\underline{I}}_{s}$ and $\underline{\underline{\mathrm{e}}}_{s y}\left(\underline{\xi}^{\alpha}\right)=y_{\alpha} \underline{\underline{I}}_{s}$. Hence

$$
\underline{\underline{\sigma}}_{s}^{e}=2 \mu \underline{\underline{\mathrm{e}}}_{s y}\left[\underline{u}^{e}+v\left(\underline{y} U_{1, x_{1}}^{1}-\underline{\xi}^{\alpha} U_{\alpha, x_{1} x_{1}}^{0}\right)\right]+\lambda \operatorname{div}_{y}\left[\underline{u}^{e}+v\left(\underline{y} U_{1, x_{1}}^{1}-\underline{\xi}^{\alpha} U_{\alpha, x_{1} x_{1}}^{0}\right) \underline{\underline{I}}_{s}\right.
$$

from which we deduce that the solution reads

$$
\underline{u}^{e}=-v\left(\underline{y} U_{1, x_{1}}^{1}-\underline{\xi}^{\alpha} U_{\alpha, x_{1} x_{1}}^{0}\right)+\underline{U}^{2}\left(x_{1}\right)+\Omega^{1}\left(x_{1}\right) \underline{a}_{1} \times \underline{y}
$$

where $\underline{U}^{2}\left(x_{1}\right)+\Omega^{1}\left(x_{1}\right) \underline{a}_{1} \times \underline{y}$ stands for any rigid in-plane translation and rotation of the section. By construction, the classical stress-strain state in elastic beam (e.g. Trabucho and Viano, 1996) is recovered

$$
\begin{aligned}
& \underline{\underline{\mathrm{e}}}_{\mathrm{sy}}\left(\underline{u}^{e}\right)=-v \underline{\underline{I}}_{\mathrm{s}} u_{1, x_{1}}^{1}, \quad \mathrm{e}_{\mathrm{n}}^{e}=u_{1, x_{1}}^{1}, \quad \text { thus } \underline{\underline{\mathrm{e}}}^{e}=\left[\underline{a}_{1} \otimes \underline{a}_{1}-v \underline{\underline{I}}_{\mathrm{s}}\right] u_{1, x_{1}}^{1} \\
& \underline{\underline{\sigma}}_{\mathrm{s}}^{e}=0, \quad \sigma_{\mathrm{n}}^{e}=E u_{1, x_{1}}^{1}=E\left(-\underline{y} \cdot U_{, x_{1} x_{1}}^{0}+U_{1, x_{1} x_{1}}^{1}\right) \quad \text { thus } \underline{\underline{\sigma}}^{e}=\sigma_{\mathrm{n}}^{e} \underline{a}_{1} \otimes \underline{a}_{1}
\end{aligned}
$$

The problem governing the poroelastic contribution $\left(\underline{\widehat{u}}^{2}, p^{1}\right)$, using the fact that $\operatorname{div}_{y}\left(\underline{u}^{2}\right)+u_{1, x_{1}}^{1}=\operatorname{div}_{y}\left(\widehat{\widehat{u}}^{2}\right)+(1-2 v) u_{1, x_{1}}^{1}$, reads

$$
\begin{aligned}
& \underline{\underline{\underline{\Sigma}}}_{\mathrm{s}}^{1}=2 \mu \underline{\underline{e}}_{s y}\left(\underline{\widehat{u}}^{2}\right)+\lambda\left[\operatorname{div}_{y}\left(\underline{\widehat{u}}^{2}\right)\right]_{\underline{I}_{s}}-\alpha p^{1} \underline{\underline{I}}_{\mathrm{s}}, \quad \underline{\operatorname{div}}_{y}\left(\underline{\underline{\Sigma}}_{\mathrm{s}}^{1}\right)=\underline{0} \text { in } S \\
& \underline{\underline{\widehat{\Sigma}}}_{\mathrm{s}}^{1} \cdot \underline{n}=\underline{0} \quad \text { on } \Gamma \\
& \operatorname{div}_{y}\left(\frac{\mathcal{K}}{i \omega \eta} \underline{\operatorname{grad}}_{y}\left(p^{1}\right)\right)-\frac{1}{M} p^{1}-\alpha \operatorname{div}_{y}\left(\widehat{\underline{u}}^{2}\right)=(1-2 v) \alpha u_{1, x_{1}}^{1} \quad \text { in } S \\
& \frac{\xi}{h} \cdot p^{1}+\underline{\operatorname{grad}}\left(p^{1}\right) \cdot \underline{n}=0 \text { on } \Gamma
\end{aligned}
$$


This is a plane poroelastic problem where the term $(1-2 v) \propto u_{1, x_{1}}^{1}$ acts as a forced volume injection. Note first that, because of the zero divergence of $\underline{\underline{\Sigma}}_{s}$ :

$$
0=\int_{S} y_{\beta}^{m} \underline{\operatorname{div}}_{y}\left(\underline{\underline{\underline{\Sigma}}}_{s}^{1}\right) d s=\int_{\Gamma} y_{\beta}^{m} \underline{\underline{\Sigma}}_{s}^{1} \cdot \underline{n} d s-m\left[\int_{S} y_{\beta}^{m-1} \underline{\underline{\underline{\Sigma}}}_{s \alpha \beta}^{1} d s\right] \underline{a}_{\alpha}
$$

then, from the free boundary condition, taking $m=1$ or 2 , we deduce

$$
\int_{S} \underline{\underline{\widehat{\Sigma}}}_{s}^{1} d s=\underline{\underline{0}}, \quad \int_{S} y_{\beta} \underline{\underline{\underline{\Sigma}}}_{s}^{1} d s=\underline{\underline{0}} \quad \text { hence } \int_{S} \operatorname{Tr}\left(\underline{\underline{\underline{\Sigma}}}_{s}^{1}\right) d s=0, \quad \int_{S} y_{\beta} \operatorname{Tr}\left(\underline{\underline{\underline{\Sigma}}}_{s}^{1}\right) d s=0
$$

and, as a consequence of the zero mean isotropic part of $\underline{\underline{\underline{\Sigma}}}_{s}^{1}$ :

$$
(\lambda+\mu) \int_{S} \operatorname{div}_{y}\left(\underline{\widehat{u}}^{2}\right) d s=\alpha \int_{S} p^{1} d s \text { therefore }(\lambda+\mu) O\left(\operatorname{div}_{y}\left(\widehat{\widehat{u}}^{2}\right)\right)=\alpha O\left(p^{1}\right)
$$

By linearity, the general solution $\left(\widehat{\underline{u}}^{2}, p^{1}\right)$ is built from the three particular solutions related to the forcing terms $-\underline{y} \cdot \underline{U}_{, x_{1}}^{0} x_{1}$ and $U_{1, x_{1}}^{1}$. Rewriting the fields as $\left(\underline{\widehat{u}}^{2}=(A /(\lambda+\mu)) \underline{\widehat{u}}, p^{1}=A p\right)$ (recall that $1 / A=1 / M+\alpha^{2} /(\lambda+\mu)$ ), leads to the following dimensionless form of the problem:

$$
\begin{aligned}
& {\underline{\operatorname{grad}_{\mathrm{y}}}}\left(\operatorname{div}_{\mathrm{y}}(\underline{\widehat{u}})\right)+(1-2 v) \Delta_{y}(\underline{\widehat{u}})-\alpha \underline{\operatorname{grad}}(p)=\underline{0} \text { in } S \\
& 2\left[(1-2 v) \underline{\mathrm{e}}_{s y}(\widehat{\widehat{u}})+v \operatorname{div}_{y}(\underline{\widehat{u}}) \underline{\underline{I}} \mathrm{~s}\right] \cdot \underline{n}-\alpha p \underline{n}=\underline{0} \text { on } \Gamma \\
& \frac{\mathcal{K} A}{i \omega \eta} \Delta_{y}(p)-\frac{A}{M} p-\alpha \frac{A}{\lambda+\mu} \operatorname{div}_{\mathrm{y}}(\underline{\widehat{u}})=V \quad \text { in } S \\
& \frac{\xi}{h} \cdot p+\underline{\operatorname{grad}}(p) \cdot \underline{n}=0 \quad \text { on } \Gamma
\end{aligned}
$$

Denoting the particular solutions $(\underline{\underline{u}}, p)$ of $(64)-(67)$ by

( $\underline{c}, \zeta)$ for $V=1$ (compression),

- $\left(\underline{b}^{\alpha}, \psi^{\alpha}\right)$ for $V=-y_{\alpha}$ (bending in direction $\left.\underline{a}_{\alpha}\right)$,

the solution $\left(\underline{\widehat{u}}^{2}, p^{1}\right)$ takes the form:

$$
\begin{aligned}
& \widehat{\underline{u}}^{2}=(1-2 v) \alpha\left[\underline{b}^{\alpha} U_{\alpha, x_{1} x_{1}}^{0}+\underline{c} U_{1, x_{1}}^{1}\right] \frac{A}{\lambda+\mu} \\
& p^{1}=(1-2 v) \alpha\left[\psi^{\alpha} U_{\alpha, x_{1} x_{1}}^{0}+\zeta U_{1, x_{1}}^{1}\right] A
\end{aligned}
$$

According to (62) we have the following identities:

$$
\begin{aligned}
& \int_{S} \operatorname{div}_{y}\left(\underline{b}^{\alpha}\right) d s=\alpha \int_{S} \psi^{\alpha} d s ; \quad \int_{S} y_{\alpha} \operatorname{div}_{y}\left(\underline{b}^{\alpha}\right) d s=\alpha \int_{S} y_{\alpha} \psi^{\alpha} d s \\
& \int_{S} \operatorname{div}_{y}(\underline{c}) d s=\alpha \int_{S} \zeta d s ; \quad \int_{S} y_{\alpha} \operatorname{div}_{y}(\underline{c}) d s=\alpha \int_{S} y_{\alpha} \zeta d s
\end{aligned}
$$

By construction, $\left(\underline{b}^{\alpha}, \psi^{\alpha}\right)$ and $(\underline{c}, \zeta)$ are complex valued and depend on (i) the geometry of the section, (ii) the dimensionless elastic parameters, $v, \alpha, \alpha^{2} M /(\lambda+\mu)$, (iii) the leakage coefficient $\xi$, and (iv) the dimensionless frequency $\omega \eta h^{2} / \mathcal{K} A$. Moreover, when $\omega \eta h^{2} / \mathcal{K} A=O(1)$, then $(\underline{c}, \zeta)$ are $\mathrm{O}(1)$ while $\left(\underline{b}^{\alpha}, \psi^{\alpha}\right)$ are $\mathrm{O}(\mathrm{h})$. Note also that for bi-symmetric section:

$$
\begin{aligned}
& b_{\beta}^{\alpha}\left(y_{2}, y_{3}\right)=(-1)^{\alpha+\beta} b_{\beta}^{\alpha}\left(-y_{2}, y_{3}\right)=(-1)^{\alpha+\beta} b_{\beta}^{\alpha}\left(y_{2},-y_{3}\right) \\
& c_{\alpha}\left(y_{2}, y_{3}\right)=-c_{\alpha}\left(-y_{2}, y_{3}\right)=c_{\alpha}\left(y_{2},-y_{3}\right), \quad \text { idem for } \psi^{\alpha} \\
& \zeta\left(y_{2}, y_{3}\right)=\zeta\left(-y_{2}, y_{3}\right)=\zeta\left(y_{2},-y_{3}\right)
\end{aligned}
$$

\section{A.2. Warping and torsion}

We focus here on the field $u_{1}^{3}$ and the expression of $M_{1}^{5}$.

$$
\begin{aligned}
& \Sigma_{\mathrm{n}, x_{1}}^{1}+\operatorname{div}_{y}\left(\underline{\sigma}_{\mathrm{t}}^{2}\right)=0 \text { in } S, \quad \underline{\sigma}_{\mathrm{t}}^{2}=\mu\left[u_{1, y_{\alpha}}^{3}+u_{\alpha, x_{1}}^{2} \underline{a}_{\alpha}\right. \\
& \underline{\sigma}_{\mathrm{t}}^{2} \cdot \underline{n}=0 \quad \text { on } \Gamma
\end{aligned}
$$

Thus $u_{1}^{3}$ obeys a linear problem and the solution is the sum of the contribution of each forcing term introduced by $\Sigma_{\mathrm{n}, x_{1}}^{1}$, and $\underline{u}_{, x_{1}}^{2}$, namely $U_{\alpha, x_{1}}^{2}, \Omega_{, x_{1}}^{1}, U_{1, x_{1} x_{1}}^{1}, U_{\alpha, x_{1} x_{1} x_{1}}^{0}$ (and the local particular fields associated to the two latter terms). 
The problem related to $U_{\alpha, x_{1}}^{2}$ is identical to that treated previously for determining $U_{1}^{1}$ and the solution is $-\underline{U}_{, x_{1}}^{2} \cdot \underline{y}$.

The problem related to $\Omega_{, x_{1}}^{1, x_{1}}$ is new. The solution reads $w(\underline{y}) \Omega^{1}\left(x_{1}\right)_{, x_{1}}$ where the warping function $w(y)$ is solution of (the zero mean value condition provides the unicity):

$$
\begin{aligned}
& \Delta_{y}(w)=0 \quad \text { in } S \\
& \left(\underline{\operatorname{grad}}_{y}(w)+\underline{a}_{1} \times \underline{y}\right) \cdot \underline{n}=0 \text { on } \Gamma, \quad \int_{S} w d s=0
\end{aligned}
$$

The problems related to $U_{1, x_{1} x_{1}}^{1}$ and $U_{\alpha, x_{1} x_{1} x_{1}}^{0}$ introduce respectively particular solutions $\varpi$ and $\chi_{\alpha}$. If the section is bisymmetric the following properties can be established from the problems set on the section:

$$
\chi_{\alpha}\left(-y_{\alpha}, y_{\beta}\right)=-\chi_{\alpha}\left(y_{\alpha}, y_{\beta}\right) ; \quad \chi_{\alpha}\left(y_{\alpha},-y_{\beta}\right)=\chi_{\alpha}\left(y_{\alpha}, y_{\beta}\right) ; \quad \text { idem for } \varpi
$$

The expression of $M_{1}^{5}$ is derived by replacing $\underline{\sigma}_{\mathrm{t}}^{2}$ by its expression:

$$
M_{1}^{5}=\int_{S} \epsilon_{1 \alpha \beta} y_{\alpha} \sigma_{\mathrm{t} \beta}^{2} d s
$$

Thus in general, there is a coupling between the torsion, bending and compression mechanisms. However, if the section is bi-symmetric, due to the resulting symmetry of the local particular fields, the only remaining terms are associated to torsion and reads

$$
M_{1}^{5}=\Omega_{, x_{1}}^{1} \mu \int_{S} \epsilon_{1 \alpha \beta} y_{\alpha}\left[w_{y_{\beta}}+\left(\underline{a}_{1} \times \underline{y}\right)_{\beta}\right] d s=\Omega_{, x_{1}}^{1} \mu \int_{S}\left(\epsilon_{1 \alpha \beta} y_{\alpha} w_{y_{\beta}}+y_{2}^{2}+y_{3}^{2}\right) d s
$$

Denoting by $I_{t}^{\prime}$ the torsion inertia that accounts for wrapping, the torsion law finally reads

$$
M_{1}^{5}=\Omega_{, x_{1}}^{1} \mu I_{t}^{\prime} ; \quad I_{t}^{\prime}=\int_{S}\left(\epsilon_{1 \alpha \beta} y_{\alpha} w_{, y_{\beta}}+y_{2}^{2}+y_{3}^{2}\right) d s
$$

A.3. Poroelastic stress-strain state with visco-inertial inner flow

In the case of inner visco-inertial flow, the problem governing $\left(\underline{\tilde{u}}^{2}, \tilde{p}^{1}\right)$ reads

$$
\begin{aligned}
& \underline{\underline{\tilde{\Sigma}}}_{\mathrm{s}}^{1}=2 \mu \underline{\underline{\mathrm{e}}}_{s y}\left(\underline{\tilde{u}}^{2}\right)+\lambda\left[\operatorname{div}_{y}\left(\underline{\tilde{u}}^{2}\right)\right] \underline{\underline{I}}_{\mathrm{s}}-\alpha p^{1} \underline{\underline{I}}_{\mathrm{s}}, \quad \underline{\operatorname{div}}_{y}\left(\underline{\underline{\tilde{\Sigma}}}_{\mathrm{s}}^{1}\right)=-\frac{i \omega \rho_{\mathrm{f}} \mathrm{K}}{\eta} \underline{\operatorname{grad}}_{y}\left(\tilde{p}^{1}\right) \text { in } S \\
& \underline{\underline{\tilde{\Sigma}}}_{\mathrm{s}}^{1} \cdot \underline{n}=0 \text { on } \Gamma \\
& \operatorname{div}_{y}\left(\frac{\mathrm{K}}{i \omega \eta} \underline{\operatorname{grad}}_{y}\left(\tilde{p}^{1}\right)\right)-\frac{1}{M} \tilde{p}^{1}-\tilde{\alpha} \operatorname{div}_{y}\left(\underline{\tilde{u}}^{2}\right)=(1-2 v) \tilde{\alpha} u_{1, x_{1}}^{1} \quad \text { in } S \\
& \underline{\xi} \cdot \tilde{p}^{1}+\underline{\operatorname{grad}}\left(\tilde{p}^{1}\right) \cdot \underline{n}=0 \quad \text { on } \Gamma
\end{aligned}
$$

This is a plane poroelastic problem where $\underline{\underline{\tilde{\Sigma}}}_{\mathrm{s}}^{1}$ is not divergence free and $(1-2 v) \tilde{\alpha} u_{1, x_{1}}^{1}$ is a forced volume injection. It takes the following dimensionless form:

$$
\begin{aligned}
& \underline{\operatorname{grad}}_{\mathrm{y}}\left(\operatorname{div}_{\mathrm{y}}(\underline{u})\right)+(1-2 v) \Delta_{y}(\underline{u})-\tilde{\alpha} \operatorname{grad}(p)=0 \text { in } S \\
& 2\left[(1-2 v) \underline{\underline{\mathrm{e}}}_{s y}(\underline{u})+v \operatorname{div}_{y}(\underline{u}) \underline{\underline{I}} \mathrm{~s}\right] \cdot \underline{n}-\alpha p \underline{n}=0 \quad \text { on } \Gamma \\
& \frac{\mathrm{KA}}{i \omega \eta} \Delta_{y}(p)-\frac{A}{M} p-\tilde{\alpha} \frac{A}{\lambda+\mu} \operatorname{div}_{\mathrm{y}}(\underline{u})=V \quad \text { in } S \\
& \frac{\xi}{h} \cdot p+\underline{\operatorname{grad}}(p) \cdot \underline{n}=0 \quad \text { on } \Gamma
\end{aligned}
$$

Denoting the particular solutions $(\underline{u}, p)$ of $(72)-(75)$ by

- $(\underline{\tilde{c}}, \tilde{\zeta})$ for $V=1$ (compression),

- $\left(\underline{\tilde{b}}^{\alpha}, \tilde{\psi}^{\alpha}\right)$ for $V=-y_{\alpha}$ (bending in direction $\underline{a}_{\alpha}$ ),

the solution $\left(\underline{\tilde{u}}^{2}, \tilde{p}^{1}\right)$ takes the form:

$$
\begin{aligned}
& \underline{\tilde{u}}^{2}=(1-2 v) \tilde{\alpha}\left[\underline{\tilde{b}}^{\alpha} U_{\alpha, x_{1} x_{1}}^{0}+\underline{\tilde{c}} U_{1, x_{1}}^{1}\right] \frac{A}{\lambda+\mu} \\
& \tilde{p}^{1}=(1-2 v) \tilde{\alpha}\left[\tilde{\psi}^{\alpha} U_{\alpha, x_{1} x_{1}}^{0}+\tilde{\zeta} U_{1, x_{1}}^{1}\right] A
\end{aligned}
$$


Hence the poroelastic contribution for the strain and effective stress tensors reads

$$
\begin{aligned}
& \underline{\underline{\tilde{e}}}^{1}=(1-2 v) \tilde{\alpha} \frac{A}{\lambda+\mu}\left[\underline{\underline{\mathrm{e}}}_{\mathrm{s}}\left(\underline{\tilde{b}}^{\alpha}\right) U_{\alpha, x_{1} x_{1}}^{0}+\underline{\underline{\mathrm{e}}}_{\mathrm{s}}(\underline{\tilde{c}}) U_{1, x_{1}}^{1}\right] \\
& \left.\underline{\underline{\tilde{\sigma}}}^{1}=(1-2 v) \tilde{\alpha} \frac{A}{\lambda+\mu}\left[\lambda\left\{\operatorname{div}_{y}\left(\underline{\tilde{b}}^{\alpha}\right) U_{\alpha, x_{1} x_{1}}^{0}+\operatorname{div}_{y}(\underline{\tilde{c}}) U_{1, x_{1}}^{1}\right)\right\} \underline{\underline{\mathrm{I}}}++2 \mu\left\{\underline{\underline{\mathrm{e}}}_{\mathrm{s}}\left(\underline{\tilde{b}}^{\alpha}\right) U_{\alpha, x_{1} x_{1}}^{0}+\underline{\underline{\mathrm{e}}}_{\mathrm{s}}(\underline{\tilde{c}}) U_{1, x_{1}}^{1}\right\}\right]
\end{aligned}
$$

that gives the total normal stress:

$$
\Sigma_{n}^{1}=\sigma_{n} 1 e-\tilde{\alpha}(1-2 v) A\left[\left[\alpha \tilde{\psi}^{\alpha}+2 v \operatorname{div}_{y}\left(\underline{\tilde{b}}^{\alpha}\right)\right] U_{\alpha, x_{1} x_{1}}^{0}+[\alpha \tilde{\zeta}+2 v \operatorname{div} y(\underline{\tilde{c}})] U_{1, x_{1}}^{1}\right]
$$

Considering bi-symmetric sections and observing that the local fields respect similar symmetries to (70), we deduce the normal and bending laws (in the usual unscaled form):

$$
\begin{aligned}
& N=\tilde{E S} \frac{\partial U_{1}}{\partial x_{1}}, \quad \tilde{E S}=E|S|-(1-2 v) \tilde{\alpha} A \int_{S}\left[\alpha \tilde{\zeta}+2 v \operatorname{div}_{y}(\underline{\tilde{c}})\right] d s \\
& M_{\alpha}=-E \tilde{I}_{\alpha} \frac{\partial^{2} U_{\alpha}}{\partial x_{1}^{2}}, \quad E \tilde{I}_{\alpha}=E I_{\alpha}+(1-2 v) \tilde{\alpha} A \int_{S} x_{\alpha}\left[\alpha \tilde{\psi}^{\alpha}+2 v \operatorname{div}_{y}\left(\underline{\tilde{b}}^{\alpha}\right)\right] d S
\end{aligned}
$$

For simplicity, the terms $\operatorname{div}_{y}\left(\underline{\tilde{b}}^{\alpha}\right), \operatorname{div}_{y}(\underline{\tilde{c}})$ are kept, although they could be re-expressed respectively with grad $\underline{\tilde{\psi}}\left(\tilde{\psi}^{\alpha}\right)$ and $\underline{\operatorname{grad}}_{y}(\tilde{\zeta})$.

\section{References}

Abramowitz, M., Stegun, I.A., 1964. Handbook of Mathematical Functions. Dover Publication Inc, New York.

Auriault, J.-L., 1980. Dynamic behaviour of a porous medium saturated by a Newtonian fluid. Int. J. Eng. Sci. 18, $775-795$.

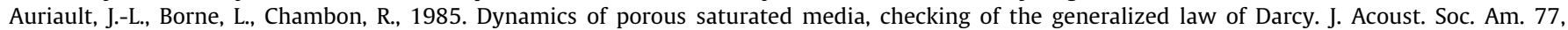
$1641-1650$.

Auriault, J.-L., Boutin, C., Geindreau, C., 2009. Homogenization of Coupled Phenomena in Heterogenous Media. ISTE and Wiley.

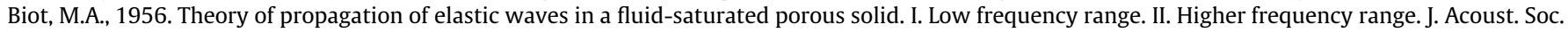
Am. 28 (2), 168-191.

Boehler, J.P., 1987. Application of Tensor Functions in Solid Mechanics. CISM Courses and Lectures, vol. 292. Springer-Verlag, Wien, New York.

Boutin, C., Auriault, J.L., 1990. Dynamic behavior of porous media saturated by a viscoelastic fluid. Int. J. Eng. Sci. 28 (11), $1157-1181$.

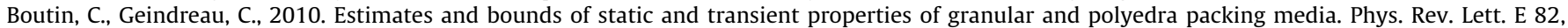
03613(18).

Boutin, C., Soubestre, J., 2011. Generalized bending continua for linear fiber reinforced materials. Int. J. Solids Struct. 48 (3), 517-534.

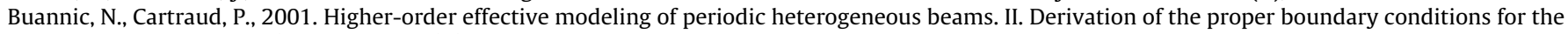
interior asymptotic solution. Int. J. Solids Struct. 38, 7168-7180.

Coussy, O., 2004. Poromechanics. Wiley, West Sussex, England.

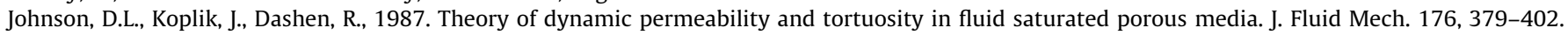

Li, L.P., Schulgasser, K., Cederbaum, G., 1995. Theory of poroelastic beams with axial diffusion. J. Mech. Phys. Solids 43 (12), $2023-2042$.

Panasenko, G., 2000. Method of asymptotic partial decomposition of rod structures. Int. J. Comput. Civil Struct. Eng. 1, 57-70.

Sanchez-Palencia, E., 1980. Non Homogeneous Media and Vibration Theory. Springer-Verlag, Berlin.

Scherer, G.W., 1992. Bending of gel beam: method for characterizing elastic properties and permeability. J. Non-Cryst. Solids Struct. $142,18-35$.

Scherer, G.W., Prevost, J.H., Wang, Z.H., 2009. Bending of a poroelastic beam with lateral diffusion. Int. J. Solids Struct. 46, $3451-3462$.

Spiegel, M.R., 1965. Laplace transforms. In: Schaum Outline Series. Mc-Graw-Hill, Berlin.

Taber, L.A., 1992. A theory for transverse deflection of poroelastic plates. J. Appl. Mech. 59 (3), 628.

Trabucho, L., Viano, J.L., 1996. Mathematical Modelling of Rod. North-Holland, Amsterdam.

Zhang, D., Cowin, S.C., 1994. Oscillatory bending of a poroelastic beam. J. Mech. Phys. Solids 42 (10), $1575-1599$. 